\title{
Clumpiness of dark matter and the positron annihilation signal
}

\author{
J. Lavalle ${ }^{1}$, J. Pochon ${ }^{2}$, P. Salati ${ }^{3,4}$, and R. Taillet ${ }^{3,4}$ \\ 1 Centre de Physique des Particules CPPM, CNRS-IN2P3/Université de la Méditerranée, 13288 Marseille, France \\ e-mail: taillet@lapp.in2p3.fr \\ 2 Laboratoire de Physique des Particules LAPP, 74941 Annecy-le-Vieux, France \\ 3 Université de Savoie, 73011 Chambéry, France \\ ${ }^{4}$ Laboratoire de Physique Théorique LAPTH, 74941 Annecy-le-Vieux, France
}

Received 29 March 2006 / Accepted 20 August 2006

ABSTRACT

\begin{abstract}
Context. The small-scale distribution of dark matter in galactic halos is poorly known. Several studies suggest that it could be very clumpy, which is of paramount importance when investigating the annihilation signal from exotic particles (e.g. supersymmetric or Kaluza-Klein).

Aims. We focus on the annihilation signal in positrons. We estimate the associated uncertainty, that is due to the fact that we do not know exactly how the clumps are distributed in the Galactic halo.

Methods. We perform a statistical study based on analytical computations, as well as numerical simulations. We study the average and variance of the annihilation signal over many Galactic halos having the same statistical properties.

Results. We find that the so-called boost factor used by many authors should be used with caution, as i) it depends on energy and ii) it may be different for positrons, antiprotons and gamma rays, a fact which has not been discussed before. As an illustration, we use our results to discuss the positron spectrum measurements by the HEAT experiment.
\end{abstract}

Key words. cosmology: dark matter - Galaxy: halo - ISM: cosmic rays

\section{Introduction}

Most observations of cosmological interest can be accounted for by assuming that our Universe contains a large amount of non-baryonic matter, usually referred to as dark matter. The mean density of matter $\Omega_{\mathrm{m}}$ can be consistently estimated to be $\Omega_{\mathrm{m}} \sim 0.23$ from many observations, whereas the baryonic density $\Omega_{\mathrm{b}}$ inferred from primordial nucleosynthesis, from Cosmic Microwave Background (CMB) anisotropies (Spergel et al. 2006), Large Scale Structures and by direct observations of luminous matter is an order of magnitude lower, namely $\Omega_{\mathrm{b}} h^{2} \approx 0.0223$.

Among the several possible solutions to the dark matter problems, the hypothesis that it could be made of a weakly interacting fundamental particle of a new kind (hereafter wimp for weakly interactive massive particle) has received considerable attention. This is partly due to the fact that this hypothesis can be tested experimentally. In particular, the detection of the annihilation products of such exotic particles would be a great achievement, and an important fraction of the astroparticle physics community is involved in that quest.

However, assuming that wimps actually do exist (see e.g. Bertone et al. 2004 for a nice review on dark matter), their nature is unknown. Some constraints can be inferred from high precision cosmological observations such as the CMB, but several particle physics models provide candidates whose properties are consistent with these observations. Extensions of the standard model of particle physics, such as supersymmetry and KaluzaKlein theories, naturally offer such candidates. The lack of information about the nature of the wimp may translate into a very large uncertainty on its spatial distribution. There are two main physical reasons for that.
First, the gravitational collapse of primordial density inhomogeneities that leads to the formation of cosmic structures is characterized by a small scale cut-off, due to several physical effects. Particles enduring collapse may interact elastically with other species or between themselves, which is responsible for diffusion. After their interactions become negligible, they become free to move out of the collapsing region: this is known as free-streaming. A general discussion of these effects can be found in Berezinsky et al. (2003). The resulting cut-off may strongly depend on the nature and properties of the wimp (see e.g. Boehm et al. 2001; Hofmann et al. 2001). For instance, the recent study by Profumo et al. (2006) gives protohalo masses ranging from $3 \times 10^{-9} M_{\odot}$ to $3 \times 10^{-1} M_{\odot}$.

Then, the structures evolve, merge and can be partially disrupted by tidal forces, so that the current cut-off in the spectrum of clump masses corresponds to the smallest surviving clumps. The situation is still unclear, as numerical simulations by Diemand et al. (2005) showed that clumps as small as $10^{-6} M_{\odot}$ could survive disruption, while analytical work by Berezinsky et al. (2006) showed that structures smaller than $10^{3} M_{\odot}$ were disrupted. The possibility that tidal interaction with stars may play an important role has also been hotly debated (Zhao et al. 2005b; Moore et al. 2005; Zhao et al. 2005a).

Different experts provide very different descriptions of the clumpiness of galactic halo. Here, we consider the wide range of possibilities as the starting point of our analysis. The amount of clumpiness is of paramount importance as it enhances the annihilation rate of wimps and increases the detection prospects.

In most studies, clumpiness is taken into account by a general, energy-independent multiplicative number called the boost factor, by which the signal computed from a smooth dark matter distribution should be multiplied. This is not correct and we 
show in this paper that the effects of clumpiness cannot be described by such a unique number. Moreover, this is a stochastic problem, in the following sense: general hypotheses about the statistical properties of the distribution of clumps in the Galactic halo can be made, but the exact position of every clump is unknown. In some cases, the expected signal from a given type of wimp can be quite sensitive to the precise position of the Earth relative to the nearest clumps.

The aim of this paper is to study the effect of halo clumpiness on the annihilation signal, focusing on the case of positrons. Taking advantage of analytical computations and numerical simulations, we investigate the statistical properties of the annihilation signal. We show that, at variance with the assumptions of most studies, the clumpiness factor depends on energy and is not the same for positrons as for gamma rays. We also show that even if the average properties (averaging being meant over a large number of realizations of our Galactic halo) of a clumpy halo may be well described by the usual boost factor, the deviations from this average may be very large and the ability to predict a signal from a model may be consequently reduced.

The importance of clumpiness in determining the dark matter annihilation signal in positrons has been assessed by Baltz \& Edsjö (1999), and further studied in Hooper et al. (2004) and Hooper \& Kribs (2004). The possibility that the positron excess observed by HEAT could be due to a single nearby clump had been raised. The probability of such a situation was estimated to be low (about $10^{-4}$ ). More recently, this proposal resurfaced (Cumberbatch \& Silk 2006). As an illustration of the methods developed in this work, we show that the odds for such an occurrence are even lower than Hooper et al's estimations.

\section{The effective boost factor}

We first consider the case of wimps annihilating into positrons and electrons at a given energy - the source spectrum of positrons can be considered monoenergetic. In Kaluza-Klein inspired models (Servant \& Tait 2003), dark matter species may substantially annihilate into electron-positron pairs with a branching ratio as large as $\sim 20 \%$. The positron production rate $P_{\mathrm{e}^{+}}$counts the number of annihilations taking place per unit volume at some point $\boldsymbol{x}$

$P_{\mathrm{e}^{+}}(\boldsymbol{x})=\delta\left\langle\sigma_{\mathrm{ann}}\left(\chi \chi \rightarrow \mathrm{e}^{+} \mathrm{e}^{-}\right) v\right\rangle\left\{\frac{\rho(\boldsymbol{x})}{m_{\chi}}\right\}^{2}$

where the $\delta$ term is equal to $1 / 2$ for a Majorana particle, taking into account the fact that these particles are not discernible, whereas it is equal to $1 / 4$ for Dirac particles, taking into account the fact that the density of particles and antiparticles is $\rho / 2$ and not $\rho$. The contribution of the infinitesimal volume $\mathrm{d}^{3} \boldsymbol{x}$ located at point $x$ to the flux at the Earth - in units of $\mathrm{cm}^{-2} \mathrm{~s}^{-1} \mathrm{sr}^{-1} \mathrm{GeV}^{-1}$ - of the resulting positrons with energy $E$ may be expressed as

$\mathrm{d} \phi=\mathcal{S} G_{\mathrm{e}^{+}}\left(\boldsymbol{x}_{\odot}, E \leftarrow \boldsymbol{x}, E_{\mathrm{S}}\right)\left\{\frac{\rho(\boldsymbol{x})}{\rho_{0}}\right\}^{2} \mathrm{~d}^{3} \boldsymbol{x}$,

where the quantity $\mathcal{S}$ depends on the mass density of reference $\rho_{0}$ and on the specific features of the high energy physics model at stake

$\mathcal{S}=\frac{\delta}{4 \pi} v_{\mathrm{e}^{+}}(E)\left\langle\sigma_{\text {ann }}\left(\chi \chi \rightarrow \mathrm{e}^{+} \mathrm{e}^{-}\right) v\right\rangle\left\{\frac{\rho_{0}}{m_{\chi}}\right\}^{2}$.

The velocity of the positron with energy $E$ at the Earth is denoted by $v_{\mathrm{e}^{+}}$. The Green function $G_{\mathrm{e}^{+}}$is discussed in Sect. 3 .
It describes the probability that a positron produced at point $\boldsymbol{x}$ with energy $E_{\mathrm{S}}$ reaches the Earth with a degraded energy $E$. As wimps are at rest with respect to the Milky Way, the energy $E_{\mathrm{S}}$ is equal to the parent particle mass $m_{\chi}$. At this stage, we keep our discussion as general as possible. Because our formalism could easily be extended to any charged species - to antiprotons or antideuterons for instance - the positron propagator will be denoted more simply as $G(\boldsymbol{x}, E)$. The total positron flux at the Earth results from the integral over the galactic DM mass distribution $\rho(\boldsymbol{x})$

$\phi=\mathcal{S} \int_{\text {DM halo }} G(\boldsymbol{x}, E)\left\{\frac{\rho(\boldsymbol{x})}{\rho_{0}}\right\}^{2} \mathrm{~d}^{3} \boldsymbol{x}$.

Should the DM halo be smoothly distributed with mass density $\rho_{\mathrm{s}}$, the positron flux would be given by relation (4) where the wimp distribution is now described by $\rho_{\mathrm{s}}$

$\phi_{\mathrm{s}}=\mathcal{S} \int_{\text {DM halo }} G(\boldsymbol{x}, E)\left\{\frac{\rho_{\mathrm{s}}(\boldsymbol{x})}{\rho_{0}}\right\}^{2} \mathrm{~d}^{3} \boldsymbol{x}$.

In the literature, the effects of clumpiness have been accounted for by shifting the flux $\phi_{\mathrm{s}}$ upwards. The multiplicative factor is called the boost. It acts as a constant of renormalization by which the flux $\phi_{\text {s }}$ generated by a smooth DM halo should be multiplied in order to take into account the enhancement of the wimp annihilation rate inside substructures. That procedure has been widely used in the past but is shown to be wrong in the present paper. In the following we discuss the method that must be followed in order to correctly compute the signal $\phi$ at the Earth.

We assume that substructures - whose density profile inside the $i$ th clump is $\delta \rho_{i}(\boldsymbol{x})$ - float inside a smoother background with mass density $\rho_{\mathrm{s}}^{\prime}$ which is a priori different from $\rho_{\mathrm{s}}$ introduced above. The halo density $\rho$ can be written as

$\rho=\rho_{\mathrm{s}}^{\prime}+\sum_{i} \delta \rho_{i}$

and each clump has a mass

$M_{i}=\int_{i \text { th clump }} \mathrm{d}^{3} \boldsymbol{x} \delta \rho_{i}(\boldsymbol{x})$.

Because wimp annihilation involves the square of the DM mass density, the production of positrons inside the $i$ th protohalo is enhanced with respect to the situation where that substructure would be diluted in the surrounding medium. Should the latter be homogeneously spread with a mass density $\rho_{\mathrm{h}}$ (which will correspond to $f \rho_{\mathrm{s}}$ below), the enhancement would be given by the boost factor $B_{i}$ which we define as

$\int_{i \text { th clump }} \mathrm{d}^{3} \boldsymbol{x} \delta \rho_{i}^{2}(\boldsymbol{x})=M_{i} \times \rho_{\mathrm{h}} B_{i}$

That relation does not mean that the annihilation signal scales linearly with the clump mass. The boost factor $B_{i}$ takes into account the inner DM distribution so that various profiles for $\delta \rho_{i}$ can lead to very different values for $B_{i}$. The relevant quantity turns out to be the effective volume $B_{i} M_{i} / \rho_{\mathrm{h}}$. In the case of model (B) of Bertone et al. (2005) where the DM clumps have been accreted around intermediate-mass black holes, the average value for that crucial factor is $\sim 4 \times 10^{5} \mathrm{kpc}^{3}$ even if the spike radius is only $\sim 1 \mathrm{pc}$. Relation (6) translates into the positron flux at the Earth

$\phi=\phi_{\mathrm{s}}^{\prime}+\phi_{r}$, 
whose component

$\phi_{r}=\sum_{i} \varphi_{i}$

is produced by the constellation of DM protohalos that pervade the Milky Way. The signal $\phi$ at the Earth is therefore enhanced by a factor of $B \equiv \phi / \phi_{\mathrm{s}}$ with respect to the situation where the DM halo is completely smooth with mass density $\rho_{\mathrm{s}}$. Many clump distributions are possible and lead to different values for the boost $B$. The distribution inside which we are embedded is of course unique. Unfortunately, we know little about it. In order to predict the set of plausible values for the boost $B$, we are forced to consider the vast ensemble of all the possible DM substructure configurations. Our lack of knowledge limits us to deriving trends for the boost. The analysis of how $B$ is statistically distributed is postponed to Sect. 4.1. Instead we now focus on its average value $B_{\text {eff }}$ which suffices when its variance is small. To proceed, a few simplifications are helpful.

(i) We will first assume that clumps are practically point-like. This hypothesis is expected to be valid when the propagation distance is large compared to the size of the clump. As the volume of the galaxy filled by the DM substructures becomes negligible, the halo density $\rho$ becomes

$\rho=\rho_{\mathrm{s}}^{\prime}+\sum_{i} M_{i} \delta^{3}\left(\boldsymbol{x}-\boldsymbol{x}_{i}\right)$,

and the smooth component $\phi_{\mathrm{s}}^{\prime}$ of the flux is given by relation (5) where the mass density $\rho_{\mathrm{s}}$ is now replaced by $\rho_{\mathrm{s}}^{\prime}$. Moreover, the positron flux $\varphi_{i}$ which the clump located at position $\boldsymbol{x}_{i}$ yields, simplifies into

$\varphi_{i}=\mathcal{S} \frac{B_{i} M_{i}}{\rho_{0}} G_{i}$,

where $G_{i} \equiv G\left(\boldsymbol{x}_{i}, E\right)$.

(ii) The boost factor $B_{i}$ at the source should vary from one protohalo to another even if the mass $M_{i}$ is assumed constant. The inner regions of the Milky Way presumably collapsed earlier than its outskirts, dragging with them substructures whose concentrations are higher than for the galactic periphery. We could expect to have larger values of $B_{i}$ inside the solar circle. However, clumps that move near the galactic center experience strong tides that could significantly reshape them (Berezinsky et al. 2003). Clumps may partially evaporate like globular clusters that exhibit characteristic tidal tails. If that effect is dominant, the clump mass is reduced and probably the boost factor too - if the density profile of the substructure readjusts itself accordingly. It is therefore difficult to predict how the clump boost factor $B_{i}$ varies with position. To simplify the discussion, we assume that all the clumps have the same mass $M_{i} \equiv M_{\mathrm{c}}$ and the same boost factor $B_{i} \equiv B_{\mathrm{c}}$. The first hypothesis is supported by numerical simulations that indicate that the mass function of substructures is a self-similar power law of slope $\mathrm{d} n(M) / \mathrm{d} \log M \propto M^{-1}$ and is actually dominated by the lightest clumps (Diemand et al. 2005). The latter hypothesis is a priori more questionable (Zhao et al. 2005b; Moore et al. 2005; Zhao et al. 2005a; Berezinsky et al. 2006). It is nevertheless a reasonable choice insofar as the effects of a granular DM distribution on the flux of positrons will be shown to be mostly local. The actual value of $B_{i}$ should not vary much in the solar neighbourhood and we can safely consider that it is constant. The expression for the flux $\phi$ simplifies into

$\phi=\phi_{\mathrm{s}}^{\prime}+\mathcal{S} \frac{B_{\mathrm{c}} M_{\mathrm{c}}}{\rho_{0}} \sum_{i} G_{i}$.

(iii) A fraction $f$ of the total DM halo is in the form of substructures embedded inside a smooth component with mass density $\rho_{\mathrm{s}}^{\prime}$. In the intermediate-mass black hole scenario of Bertone et al. (2005), the fraction $f$ is so small that $\rho_{\mathrm{s}}^{\prime} \simeq \rho_{\mathrm{s}}$. On the contrary, in the Diemand et al. (2005) simulations, a value as large as $f \sim 0.5$ is found with a preponderance of small-scale clumps which should trace the smooth DM density as ascertained in Berezinsky et al. (2003). The mass density $\rho_{\mathrm{s}}^{\prime}$ could be quite different from $\rho_{\mathrm{s}}$ but its contribution $\phi_{\mathrm{s}}^{\prime}$ to the overall signal $\phi$ is small. We will assume for simplicity that

$\rho_{\mathrm{s}}^{\prime}=(1-f) \rho_{\mathrm{s}}$,

where $f$ is constant all over the Milky Way. The corresponding flux ratio $\phi_{\mathrm{s}}^{\prime} / \phi_{\mathrm{s}}$ - which should not exceed 1 in any case - is now given by the factor $(1-f)^{2}$.

(iv) A number $N_{\mathrm{H}}$ of DM substructures pervade the Milky Way halo. In this analysis, we will not consider the fluctuations of that number. The probability that one of those lies at point $\boldsymbol{x}$ is controlled by the distribution $p(\boldsymbol{x})$. The number of clumps that the volume $\mathrm{d}^{3} \boldsymbol{x}$ contains on average is

$\langle\mathrm{d} n\rangle=N_{\mathrm{H}} p(\boldsymbol{x}) \mathrm{d}^{3} \boldsymbol{x}$.

We infer an average flux at the Earth

$\langle\phi\rangle=(1-f)^{2} \phi_{\mathrm{s}}+\mathcal{S} \frac{B_{\mathrm{c}} M_{\mathrm{c}}}{\rho_{0}}\left\langle\sum_{i} G_{i}\right\rangle$,

where the average sum over the Green functions $G_{i}$ is given by the integral

$\left\langle\sum_{i} G_{i}\right\rangle=\int_{\text {DM halo }} G(x, E)\langle\mathrm{d} n\rangle$.

For illustration purposes, we have chosen in our numerical examples a particular clump distribution. Inspired by Diemand et al. (2005), we have assumed that protohalos trace the smooth distribution of dark matter with

$p(\boldsymbol{x})=\frac{\rho_{\mathrm{S}}(\boldsymbol{x})}{M_{\mathrm{H}}}$,

where $M_{\mathrm{H}}$ is the mass of the DM Milky Way halo. We stress that our analysis does not depend on that specific choice and is completely general. Considering a different distribution $p(\boldsymbol{x})-$ with no relation to the mass density $\rho_{\mathrm{s}}$ in particular - would not qualitatively affect the main conclusions of our analysis.

We derive the effective boost

$B_{\mathrm{eff}}(E) \equiv \frac{\langle\phi\rangle}{\phi_{\mathrm{s}}}=(1-f)^{2}+f B_{\mathrm{c}} \frac{\mathcal{I}_{1}}{\mathcal{I}_{2}}$

where the integral $\mathcal{I}_{n}$ is defined by

$\mathcal{I}_{n}(E)=\int_{\text {DM halo }} G(\boldsymbol{x}, E)\left\{\frac{\rho_{\mathrm{s}}(\boldsymbol{x})}{\rho_{0}}\right\}^{n} \mathrm{~d}^{3} \boldsymbol{x}$.

Although the boost at the source $B_{\mathrm{c}}$ is fixed, the boost of the signal at the Earth $B_{\text {eff }}$ depends on both the nature and the energy of the cosmic ray species through the Green function $G$ and the 

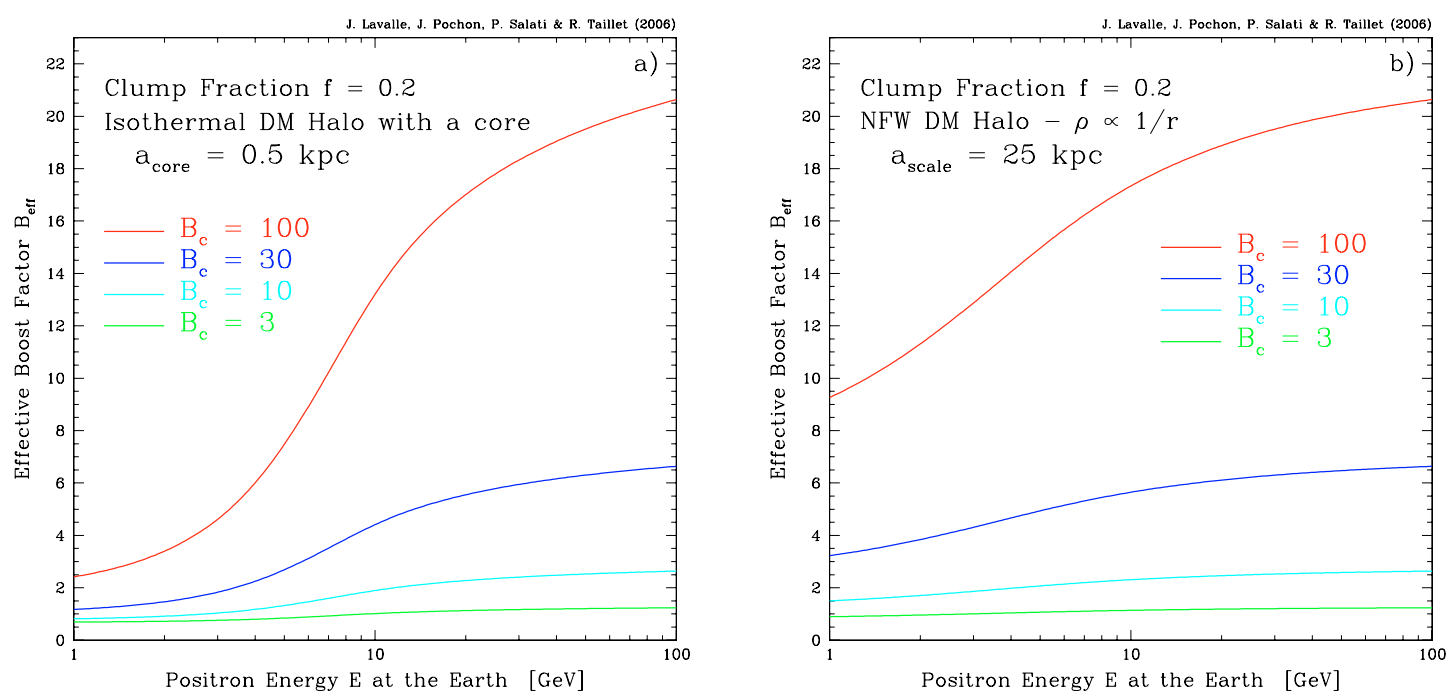

Fig. 1. The effective boost factor $B_{\text {eff }}$ as a function of the positron energy $E$ in the case of a $100 \mathrm{GeV}$ line. A fraction $f=0.2$ of the DM distribution is in the form of substructures whose individual boost factor $B_{\mathrm{c}}$ - relative to the solar neighbourhood density - has been varied from 3 to 100 . An isothermal halo - panel a) - and a NFW profile - panel b) - are considered. They illustrate the influence of the central profile index. The increase of $B_{\text {eff }}$ is noticeable especially around $E \sim 10 \mathrm{GeV}$.

integrals $\mathcal{I}_{1}$ and $\mathcal{I}_{2}$. As the flux $\phi_{\mathrm{s}}$ is shifted upwards as a result of clumpiness, it also experiences a spectral distorsion insofar as $B_{\text {eff }}$ is energy dependent. This property has never been noticed before and is illustrated in the two panels of Fig. 1 where the case of a $100 \mathrm{GeV}$ positron line is featured. A fraction $f=0.2$ of the $\mathrm{DM}$ halo has collapsed in the form of clumps whose boost factor $B_{\mathrm{c}}$ vary from 3 to 100 . In the Diemand et al. (2005) numerical simulations, such a value for the fraction would correspond to a minimum mass scale of $10^{4} M_{\odot}$. The latter lies typically at the lower tip of the range of protohalo masses which we have used in our examples. As for the boost $B_{\mathrm{c}}$, the values quoted in the literature vary from a few (Berezinsky et al. 2006) up to over two orders of magnitude (Diemand et al. 2005). The reference mass density $\rho_{0}$ has been set equal to the solar neighbourhood value of $\rho_{\mathrm{s}}(\odot)=0.3 \mathrm{GeV} \mathrm{cm}{ }^{-3}$. The increase of the effective boost factor with positron energy is clear in both panels. Near the line - in the region where $E$ tends to the input energy $E_{\mathrm{S}}$ - the positron Green function $G$ probes only a small region of the Milky Way halo around the solar system. With our definition of $\rho_{0}$, the integral ratio $\mathcal{I}_{1} / \mathcal{I}_{2}$ is unity and $B_{\text {eff }}$ may be approximated by $\sim f B_{\mathrm{c}}$. If $E$ is now varied from its upper limit $E_{\mathrm{S}}$ downwards, larger portions of the halo come into play in the integrals $\mathcal{I}_{1}$ and $\mathcal{I}_{2}$, decreasing their ratio. That effect is quite obvious in panel a) where an isothermal profile is assumed with core radius $a_{\text {core }}=0.5 \mathrm{kpc}$. The DM density $\rho_{\mathrm{s}}$ within $1 \mathrm{kpc}$ of the galactic center is larger than in the case of a NFW distribution and the relative increase of $\mathcal{I}_{2}-$ where the square of $\rho_{\mathrm{s}}$ is relevant and not merely $\rho_{\mathrm{s}}$ alone - with respect to $\mathcal{I}_{1}$ is more pronounced. It is possible that the energy dependence of $B_{\text {eff }}$ that we have discovered could strengthen the case of DM particles as a plausible explanation of the still putative positron excess reported by HEAT. In the example featured in Fig. 1, the largest spectral distortion is actually obtained for a positron energy $E \sim 10 \mathrm{GeV}$.

This distorsion effect should not be present in the case of gamma rays, whose propagation does not depend on energy. For antiprotons, the Green function already probes a significant portion of the DM halo, and we anticipate a mild dependence of the boost factor on the energy.

\section{The positron propagator}

The departures of the positron flux from $\phi_{\mathrm{s}}$ are expected to be large when the positron energy $E$ is close to the production value $E_{\mathrm{S}}$. In this regime, the particles cannot have been produced far away. They mostly originate from a region close to the solar system inside which the distribution of clumps may significantly fluctuate. That is why we have focused our analysis on cosmic ray positrons whose propagation throughout the galaxy is now briefly sketched.

The master equation for positron propagation is the continuity relation

$\partial_{\mu} J^{\mu}+\partial_{E} J^{E}=Q$

where $Q$ denotes the production rate of positrons per unit of volume and energy. The space-time vector current is defined as

$J^{\mu}=\frac{\mathrm{d} n}{\mathrm{~d} E}\left\langle\dot{x}^{\mu}\right\rangle$

The time-component $J^{0}=\mathrm{d} n / \mathrm{d} E \equiv \psi(\boldsymbol{x}, E)$ denotes the number density of particles per unit of volume and energy. The space current accounts for the scattering of cosmic rays upon the inhomogeneities of the galactic magnetic fields which is described as a diffusion process with

$\boldsymbol{J}=-K(\boldsymbol{x}, E) \boldsymbol{\nabla} \psi$.

The energy component $J^{E}$ depends on the energy loss rate $b$ through

$J^{E}=\psi\{\langle\dot{E}\rangle \equiv-b(E)\}$

Above a few $\mathrm{GeV}$, positron energy losses are dominated by synchrotron radiation in the galactic magnetic fields and by inverse Compton scattering on stellar light and on CMB photons. The energy loss rate $b$ depends on the positron energy $E$ through

$b(E)=\frac{E^{2}}{E_{0} \tau_{E}}$ 
We have set the energy of reference $E_{0}$ to $1 \mathrm{GeV}$ and the typical energy loss time is $\tau_{E}=10^{16} \mathrm{~s}$. The master Eq. (21) may be expanded into

$\frac{\partial \psi}{\partial t}-\boldsymbol{\nabla} \cdot\{K(\boldsymbol{x}, E) \boldsymbol{\nabla} \psi\}-\frac{\partial}{\partial E}\{b(E) \psi\}=Q(\boldsymbol{x}, E)$.

In order to simplify the discussion, steady state is assumed and the space diffusion coefficient $K$ is taken to be homogeneous with the energy dependence

$K(E)=K_{0}\left\{\frac{E}{E_{0}}\right\}^{\alpha}$.

The diffusion coefficient at $1 \mathrm{GeV}$ is $K_{0}=3 \times 10^{27} \mathrm{~cm}^{2} \mathrm{~s}^{-1}$ with a spectral index of $\alpha=0.6$. The master Eq. (26) simplifies into

$K_{0} \epsilon^{\alpha} \Delta \psi+\frac{\partial}{\partial \epsilon}\left\{\frac{\epsilon^{2}}{\tau_{E}} \psi\right\}+Q=0$,

where $\epsilon$ denotes the ratio $E / E_{0}$.

Equation (28) may be solved with the Baltz \& Edsjö (1999) solution of translating the energy $E$ into the pseudo-time

$\tilde{t}(E)=\tau_{E}\left\{v(E)=\frac{\epsilon^{\alpha-1}}{1-\alpha}\right\}$.

The energy losses that positrons experience lead to an evolution in this pseudo-time so that the propagation Eq. (28) greatly simplifies into

$\frac{\partial \tilde{\psi}}{\partial \tilde{t}}-K_{0} \Delta \tilde{\psi}=\tilde{Q}(\boldsymbol{x}, \tilde{t})$.

The space and energy positron density is now $\tilde{\psi}=\epsilon^{2} \psi$ whereas the positron production rate has become $\tilde{Q}=\epsilon^{2-\alpha} Q$. Notice that both $\tilde{\psi}$ and $\tilde{Q}$ have the same dimensions as before because $\epsilon$ is dimensionless. Without any space boundary condition, Eq. (30) may be readily solved. If a drop is deposited at the origin of the coordinates at pseudo-time $\tilde{t}_{\mathrm{S}}=0$

$\tilde{Q}\left(\boldsymbol{x}_{\mathrm{S}}, \tilde{t}_{\mathrm{S}}\right)=\delta^{3}\left(\boldsymbol{x}_{\mathrm{S}}\right) \delta\left(\tilde{t}_{\mathrm{S}}\right)$,

the subsequent diffusion in an infinite 3D space would result into the density $\tilde{\psi}$ at position $\boldsymbol{x}$ and pseudo-time $\tilde{t}$ given by the well-known Green function

$$
\begin{aligned}
\tilde{\psi}(\boldsymbol{x}, \tilde{t}) & \equiv \tilde{G}(\boldsymbol{x}, \tilde{t} \leftarrow \mathbf{0}, 0) \\
& =\theta(\tilde{t})\left\{4 \pi K_{0} \tilde{t}\right\}^{-3 / 2} \exp \left\{-\frac{r^{2}}{4 K_{0} \tilde{t}}\right\},
\end{aligned}
$$

where $r \equiv|x|$. The general solution of Eq. (30) may be expressed with the Green function $\tilde{G}$ as

$\tilde{\psi}(\boldsymbol{x}, \tilde{t})=\int_{\tilde{t}_{\mathrm{S}}=0}^{\tilde{t}_{\mathrm{S}}=\tilde{t}} \mathrm{~d} \tilde{t}_{\mathrm{S}} \int \mathrm{d}^{3} \boldsymbol{x}_{\mathrm{S}} \tilde{\boldsymbol{G}}\left(\boldsymbol{x}, \tilde{t} \leftarrow \boldsymbol{x}_{\mathrm{S}}, \tilde{t}_{\mathrm{S}}\right) \tilde{Q}\left(\boldsymbol{x}_{\mathrm{S}}, \tilde{t}_{\mathrm{S}}\right)$,

and translates into

$$
\begin{aligned}
\psi(\boldsymbol{x}, E)= & \int_{E_{\mathrm{S}}=E}^{E_{\mathrm{S}}=+\infty} \mathrm{d} E_{\mathrm{S}} \int \mathrm{d}^{3} \boldsymbol{x}_{\mathrm{S}} \\
& \times G_{\mathrm{e}^{+}}\left(\boldsymbol{x}, E \leftarrow \boldsymbol{x}_{\mathrm{S}}, E_{\mathrm{S}}\right) Q\left(\boldsymbol{x}_{\mathrm{S}}, E_{\mathrm{S}}\right) .
\end{aligned}
$$

The positron propagator may be obtained from $\tilde{G}$ through

$G_{\mathrm{e}^{+}}\left(\boldsymbol{x}, E \leftarrow \boldsymbol{x}_{\mathrm{S}}, E_{\mathrm{S}}\right)=\frac{\tau_{E}}{E_{0} \epsilon^{2}} \tilde{G}\left(\boldsymbol{x}, \tilde{t} \leftarrow \boldsymbol{x}_{\mathrm{S}}, \tilde{t}_{\mathrm{S}}\right)$, where the connection between the energy $E$ and the pseudotime $\tilde{t}$ is given by relation (29). In the case of monochromatic positrons, the production rate is

$Q(\boldsymbol{x}, E)=P_{\mathrm{e}^{+}}(\boldsymbol{x}) \delta\left(E-E_{\mathrm{S}}\right)$,

and the positron space and energy density at the Earth may be expressed as

$$
\begin{aligned}
\psi\left(\boldsymbol{x}_{\odot}, E\right)= & \theta\left(E_{\mathrm{S}}-E\right) \\
& \times \int \mathrm{d}^{3} x_{\mathrm{S}} G_{\mathrm{e}^{+}}\left(\boldsymbol{x}_{\odot}, E \leftarrow \boldsymbol{x}_{\mathrm{S}}, E_{\mathrm{S}}\right) P_{\mathrm{e}^{+}}\left(\boldsymbol{x}_{\mathrm{S}}\right) .
\end{aligned}
$$

Equation (2) is based on this relation.

The diffusive halo inside which cosmic rays propagate before escaping into the intergalactic medium is pictured as a flat cylinder with radius $R_{\text {gal }}=20 \mathrm{kpc}$ and extends along the vertical direction from $z=-L$ up to $z=+L$. We have assumed here a halfthickness of $L=3 \mathrm{kpc}$. Without any boundary condition, the propagator $\tilde{G}$ would be given by the $3 \mathrm{D}$ relation (32). However, cosmic rays may escape outside the diffusive halo and $\tilde{G}$ should account for that leakage. In spite of the boundary at $R_{\mathrm{gal}}$, we have assumed that cosmic ray diffusion is not limited along the radial direction but takes place inside an infinite horizontal slab with thickness $2 L$. We have nevertheless disregarded sources located at a radial distance $R$ larger than $R_{\text {gal }}$. Indeed, because their energy is rapidly degraded as they propagate, positrons are observed close to where they are produced. Our radial treatment is justified because positrons do not originate from far away (Maurin \& Taillet 2003). Even in the case of antiprotons for which the galactic propagation range is significantly larger than for positrons, the effects of the radial boundary at the Earth are not significant as cosmic ray species tend to leak above and beneath the diffusive halo at $z= \pm L$ instead of traveling a long distance along the galactic disk. The infinite slab hypothesis allows the radial and vertical directions to be disentangled in the reduced propagator $\tilde{G}$ which may now be expressed as

$$
\begin{aligned}
\tilde{G}\left(\boldsymbol{x}, \tilde{t} \leftarrow \boldsymbol{x}_{\mathrm{S}}, \tilde{t}_{\mathrm{S}}\right)= & \frac{\theta(\tilde{\tau})}{4 \pi K_{0} \tilde{\tau}} \exp \left\{-\frac{R^{2}}{4 K_{0} \tilde{\tau}}\right\} \\
& \times \tilde{V}\left(z, \tilde{t} \leftarrow z_{\mathrm{S}}, \tilde{t}_{\mathrm{S}}\right),
\end{aligned}
$$

where $\tilde{\tau}=\tilde{t}-\tilde{t}_{\mathrm{S}}$. The radial distance between the source $\boldsymbol{x}_{\mathrm{S}}$ and the point $\boldsymbol{x}$ of observation is defined as

$R=\left\{\left(x-x_{\mathrm{S}}\right)^{2}+\left(y-y_{\mathrm{S}}\right)^{2}\right\}^{1 / 2}$.

Should propagation be free along the vertical direction, the propagator $\tilde{V}$ would be given by the $1 \mathrm{D}$ solution $\mathcal{V}_{1 \mathrm{D}}$ to the diffusion Eq. (30)

$$
\begin{aligned}
\tilde{V}\left(z, \tilde{t} \leftarrow z_{\mathrm{S}}, \tilde{t}_{\mathrm{S}}\right) & \equiv \mathcal{V}_{1 \mathrm{D}}\left(z, \tilde{t} \leftarrow z_{\mathrm{S}}, \tilde{t}_{\mathrm{S}}\right) \\
& =\frac{\theta(\tilde{\tau})}{\sqrt{4 \pi K_{0} \tilde{\tau}}} \exp \left\{-\frac{\left(z-z_{\mathrm{S}}\right)^{2}}{4 K_{0} \tilde{\tau}}\right\} .
\end{aligned}
$$

But the vertical boundary conditions definitely need to be implemented. Wherever the source inside the slab, the positron density vanishes at $z= \pm L$. A first approach relies on the method of the so-called electrical images and has been implemented by Baltz \& Edsjö (1999). Any point-like source inside the slab is associated with the infinite series of its multiple images through the boundaries at $z= \pm L$ which act as mirrors. The $n$th image is located at

$z_{n}=2 L n+(-1)^{n} z_{\mathrm{S}}$, 
and has a positive or negative contribution depending on whether $n$ is an even or odd number. When the diffusion time $\tilde{\tau}$ is small, the 1D solution (40) is a quite good approximation. The relevant parameter is

$\zeta=\frac{L^{2}}{4 K_{0} \tilde{\tau}}$

and in the regime where it is much larger than 1, the propagation is insensitive to the vertical boundaries. On the contrary, when $\zeta$ is much smaller than 1, a large number of images need to be taken into account in the sum

$\tilde{V}\left(z, \tilde{t} \leftarrow z_{\mathrm{S}}, \tilde{t}_{\mathrm{S}}\right)=\sum_{n=-\infty}^{+\infty}(-1)^{n} \mathcal{V}_{1 \mathrm{D}}\left(z, \tilde{t} \leftarrow z_{n}, \tilde{\mathrm{S}}_{\mathrm{S}}\right)$,

and convergence may be a problem. A different approach is possible in that case. The 1D diffusion Eq. (30) looks like the Schrödinger equation - in imaginary time - that accounts for the behaviour of a particle inside an infinitely deep 1D potential well that extends from $z=-L$ to $z=+L$. The eigenfunctions of the associated Hamiltonian are both even

$\varphi_{n}(z)=\sin \left\{k_{n}(L-|z|)\right\}$

and odd

$\varphi_{n}^{\prime}(z)=\sin \left\{k_{n}^{\prime}(L-z)\right\}$

functions of the vertical coordinate $z$. The wave-vectors $k_{n}$ and $k_{n}^{\prime}$ are respectively defined as

$k_{n}=\left(n-\frac{1}{2}\right) \frac{\pi}{L}$ (even) and $\quad k_{n}^{\prime}=n \frac{\pi}{L}($ odd $)$.

The vertical propagator may be expanded as the series

$$
\begin{aligned}
\tilde{V}\left(z, \tilde{t} \leftarrow z_{\mathrm{S}}, \tilde{t}_{\mathrm{S}}\right)= & \sum_{n=1}^{+\infty} \frac{1}{L}\left\{\mathrm{e}^{-\lambda_{n} \tilde{\tau}} \varphi_{n}\left(z_{\mathrm{S}}\right) \varphi_{n}(z)\right. \\
& \left.+\mathrm{e}^{-\lambda_{n}^{\prime} \tilde{\tau}} \varphi_{n}^{\prime}\left(z_{\mathrm{S}}\right) \varphi_{n}^{\prime}(z)\right\},
\end{aligned}
$$

where the time constants $\lambda_{n}$ and $\lambda_{n}^{\prime}$ are respectively equal to $K_{0} k_{n}{ }^{2}$ and $K_{0} k_{n}^{\prime 2}$. In the regime where $\zeta$ is much smaller than 1 - for very large values of the diffusion time $\tilde{\tau}$ - just a few eigenfunctions need to be considered for the sum (47) to converge.

\section{An analytic approach of the cosmic ray flux fluctuations}

\subsection{The random flux $\phi_{r}$ and its variance}

The cosmic ray flux (13) at the Earth contains the random component

$\phi_{r}=\sum_{i} \varphi_{i}=\mathcal{S} \frac{B_{\mathrm{c}} M_{\mathrm{c}}}{\rho_{0}} \sum_{i} G_{i}$,

which is produced by the constellation of DM clumps inside the Milky Way halo.

(i) The actual distribution of DM substructures is unique, as is the cosmic ray flux that it generates at the Earth. We will nevertheless consider it as one particular realization among an essentially infinite number of different possible realizations. We furthermore assume that clumps are distributed at random and that the set of all their possible distributions makes up the statistical ensemble that we consider in this section. The aim of our analysis is to investigate how strongly the flux $\phi_{r}$ may fluctuate as a result of the random nature of the wimp clump distribution. We will derive the associated cosmic-ray flux variance $\sigma_{r}$ defined as

$\sigma_{r}^{2}=\left\langle\phi_{r}^{2}\right\rangle-\left\langle\phi_{r}\right\rangle^{2}$.

The variance $\sigma_{r}$ is an essential tool. Because the total flux $\phi$ and its random component $\phi_{r}$ are shifted with respect to each other by the constant quantity

$\phi-\phi_{r}=(1-f)^{2} \phi_{\mathrm{s}}$,

the variance of the former is given by

$\sigma_{\phi}^{2}=\left\langle\phi^{2}\right\rangle-\langle\phi\rangle^{2}=\left\langle\phi_{r}^{2}\right\rangle-\left\langle\phi_{r}\right\rangle^{2}=\sigma_{r}^{2}$.

The effective boost $B_{\text {eff }}$ discussed in Sect. 2 is an average value around which the true flux enhancement $B \equiv \phi / \phi_{\mathrm{s}}$ fluctuates with the variance

$\sigma_{B}=\frac{\sigma_{\phi}}{\phi_{\mathrm{s}}}=\frac{\sigma_{r}}{\phi_{\mathrm{s}}}$

Therefore, the determination of $\sigma_{r}$ leads immediately to the boost fluctuations $\sigma_{B}$.

(ii) We will furthermore assume that clumps are distributed independently of each other. The problem is then greatly simplified because we just need to determine how a single clump is distributed inside the galactic halo in order to derive the statistical properties of an entire constellation of such substructures. In particular, the average value $\left\langle\phi_{r}\right\rangle$ of the random component of the cosmic ray flux is readily obtained from the average flux $\langle\varphi\rangle$ produced by a single clump through the relation

$\left\langle\phi_{r}\right\rangle=N_{\mathrm{H}}\langle\varphi\rangle$,

where $N_{\mathrm{H}}$ denotes the total number of clumps to be considered. The variance $\sigma_{r}-$ which is the crucial quantity for the flux fluctuations - may also be expressed as

$\sigma_{r}^{2}=N_{\mathrm{H}} \sigma^{2}=N_{\mathrm{H}}\left\{\left\langle\varphi^{2}\right\rangle-\langle\varphi\rangle^{2}\right\}$.

(iii) The set of the random distributions of one single clump inside the domain $\mathcal{D}_{\mathrm{H}}$ forms the statistical ensemble $\mathcal{T}$ that we need to consider. An event from that ensemble consists of a clump located at position $\boldsymbol{x}$ within the elementary volume $\mathrm{d}^{3} \boldsymbol{x}$. Its probability $\mathrm{d} P$ will be assumed to follow the smoothed DM mass distribution $\rho_{\mathrm{s}}$ so that

$\mathrm{d} P=p(\boldsymbol{x}) \mathrm{d}^{3} \boldsymbol{x}=\frac{\rho_{\mathrm{s}}(\boldsymbol{x})}{M_{\mathrm{H}}} \mathrm{d}^{3} \boldsymbol{x}$.

The domain $\mathcal{D}_{\mathrm{H}}$ over which our statistical analysis is performed is so large that the total number $N_{\mathrm{H}}$ of clumps that it contains is essentially infinite. That region $\mathcal{D}_{\mathrm{H}}$ behaves therefore like a so-called thermostat in statistical mechanics. It encompasses the diffusive halo and may even be much bigger. It may be thought of - but not exclusively - as the entire Milky Way DM halo. Its actual size has no importance because it will disappear from the final results in the limit where the ratio $1 / N_{\mathrm{H}}$ is negligible. The only requirement is that $N_{\mathrm{H}}$ should be much larger than the typical number $N_{\mathrm{S}}$ of clumps that effectively contribute to the signal $\phi_{r}$ at the Earth. The domain $\mathcal{D}_{\mathrm{H}}$ contains the total DM mass $M_{\mathrm{H}}-\mathrm{a}$ fraction $f$ of which lies in $N_{\mathrm{H}}$ identical clumps so that

$N_{\mathrm{H}} M_{\mathrm{c}}=f M_{\mathrm{H}}$. 
We are now ready to derive the probability distribution $\mathcal{P}(\varphi)$ associated with the signal $\varphi$ that a single clump generates. The statistical properties of the random variable $\varphi\{\mathcal{T}\}$ reflect those of the statistical ensemble $\mathcal{T}$ itself. More precisely, the probability function $\mathcal{P}(\varphi)$ is related to the space distribution $p(\boldsymbol{x})$ through

$\mathcal{P}(\varphi) \mathrm{d} \varphi=\mathrm{d} P=\int_{\mathcal{D}_{\varphi}} p(\boldsymbol{x}) \mathrm{d}^{3} \boldsymbol{x}$.

The subdomain $\mathcal{D}_{\varphi}$ over which the space distribution $p(\boldsymbol{x})$ should be integrated in the previous expression yields a flux at the Earth between $\varphi$ and $\varphi+d \varphi\left(\mathcal{D}_{\mathrm{H}}\right.$ is thus the union of all $\left.\mathcal{D}_{\varphi}\right)$. In the case of positrons, the probability distribution $\mathcal{P}(\varphi)$ will be shown in Sect. 4.2 to concentrate around a flux $\varphi$ equal to 0 . The average value - over the statistical ensemble $\mathcal{T}-$ of any function $\mathcal{F}$ that depends on the flux $\varphi$ may be expressed as

$\langle\mathcal{F}\rangle=\int \mathcal{F}(\varphi) \mathcal{P}(\varphi) \mathrm{d} \varphi=\int_{\mathcal{D}_{\mathrm{H}}} \mathcal{F}\{\varphi(\boldsymbol{x})\} p(\boldsymbol{x}) \mathrm{d}^{3} \boldsymbol{x}$.

In particular, the flux that a single clump yields on average at the Earth is readily derived from the integral

$\langle\varphi\rangle=\int_{\mathcal{D}_{\mathrm{H}}} \varphi(\boldsymbol{x}) p(\boldsymbol{x}) \mathrm{d}^{3} \boldsymbol{x}=\mathcal{S} \frac{M_{\mathrm{c}} B_{\mathrm{c}}}{M_{\mathrm{H}}} \mathcal{I}_{1}$,

where $\mathcal{I}_{n}$ has been defined in relation (20). The average value of the random flux $\phi_{r}$ implies $N_{\mathrm{H}}$ clumps and expression (53) with the help of relation (56) - leads to

$\frac{\left\langle\phi_{r}\right\rangle}{\phi_{\mathrm{s}}}=\frac{\mathcal{S} f B_{\mathrm{c}} I_{1}}{\phi_{\mathrm{s}}}=f B_{\mathrm{c}} \frac{\mathcal{I}_{1}}{\mathcal{I}_{2}}$,

and to formula (19).

Starting from the definition (54), the variance $\sigma_{r}$ may be derived in the same spirit with

$\frac{\sigma_{r}^{2}}{\left\langle\phi_{r}\right\rangle^{2}}=\frac{1}{N_{\mathrm{H}}} \frac{\left\langle\varphi^{2}\right\rangle}{\langle\varphi\rangle^{2}}-\frac{1}{N_{\mathrm{H}}}$

With the help of relation (58), the mean square of the single clump flux may be expressed as

$\left\langle\varphi^{2}\right\rangle=\int_{\mathcal{D}_{\mathrm{H}}} \varphi^{2}(\boldsymbol{x}) p(\boldsymbol{x}) \mathrm{d}^{3} \boldsymbol{x}=\frac{\mathcal{S}^{2} M_{\mathrm{c}}^{2} B_{\mathrm{c}}^{2}}{\rho_{0} M_{\mathrm{H}}} \mathcal{J}_{1}$,

where the integral $\mathcal{J}_{n}$ is defined as

$\mathcal{J}_{n}(E)=\int_{\text {DM halo }} G^{2}(\boldsymbol{x}, E)\left\{\frac{\rho_{\mathrm{s}}(\boldsymbol{x})}{\rho_{0}}\right\}^{n} \mathrm{~d}^{3} \boldsymbol{x}$.

Some straightforward algebra leads to the relative variance

$\frac{\sigma_{r}^{2}}{\left\langle\phi_{r}\right\rangle^{2}}=\frac{M_{H}}{\rho_{0} N_{\mathrm{H}}} \frac{\mathcal{J}_{1}}{\mathcal{I}_{1}^{2}}-\frac{1}{N_{\mathrm{H}}} \simeq \frac{M_{\mathrm{c}}}{f \rho_{0}} \frac{\mathcal{J}_{1}}{\mathcal{I}_{1}^{2}}$.

Because the domain $\mathcal{D}_{\mathrm{H}}$ is so large - remember that both $\mathcal{D}_{\mathrm{H}}$ and the Milky Way DM halo encompasses the diffusive halo we can safely drop the ratio $1 / N_{\mathrm{H}}$ in the previous expression.

The positron propagator of Sect. 3 has been used in relation (64) to derive the solid curves of Fig. 2. At fixed $N_{\mathrm{H}}$, the clump mass $M_{\mathrm{c}}$ is determined by Eq. (56) and the relative variance $\sigma_{r} /\left\langle\phi_{r}\right\rangle$ increases with the positron energy $E$ at the Earth. This behaviour will be explained in Sect. 5 with the hard-sphere approximation. The ratio $\sigma_{r} /\left\langle\phi_{r}\right\rangle$ is proportional to $1 / \sqrt{N_{\mathrm{H}}} \propto \sqrt{M_{\mathrm{c}}}$, and weighted by an effective volume $\mathcal{J}_{1} / \mathcal{I}_{1}^{2}$. The curves are therefore shifted upwards when the clump mass

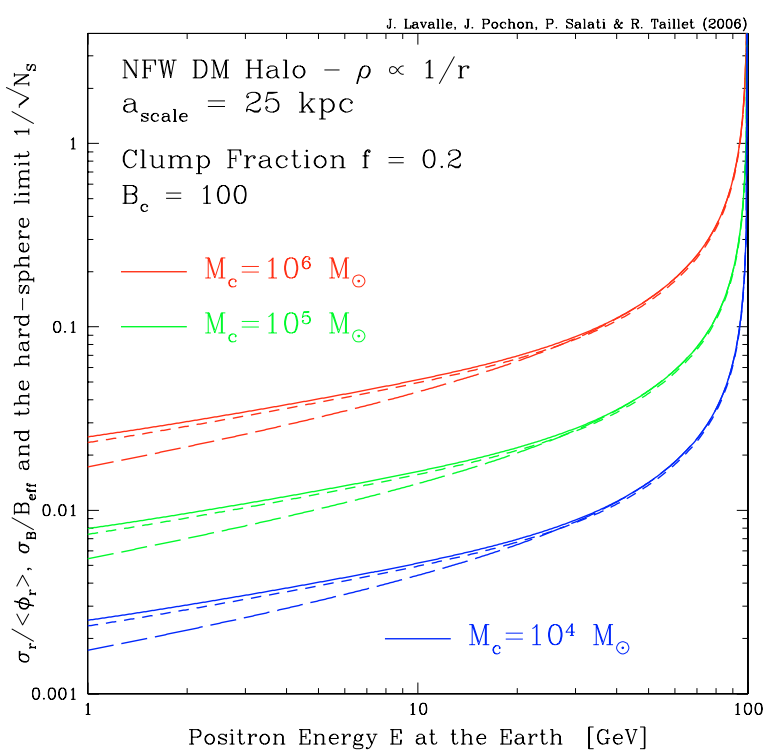

Fig. 2. The relative variance $\sigma_{r} /\left\langle\phi_{r}\right\rangle$ of the random component of the positron flux at the Earth - solid lines - and its hard-sphere approximation - long-dashed curves - as a function of the positron energy $E$ for three different values of the clump mass $M_{\mathrm{c}}$. The injected positron energy $E_{\mathrm{S}}$ has been set equal to $100 \mathrm{GeV}$. A NFW profile with typical scale $25 \mathrm{kpc}$ has been assumed. At fixed clump mass, the variance increases with $E$ and matches its hard-sphere approximation above $\sim 40 \mathrm{GeV}$. As the number of clumps is decreased, the curves are shifted upwards by a factor of $1 / \sqrt{N_{\mathrm{H}}} \propto \sqrt{M_{\mathrm{c}}}$. The relative variance $\sigma_{B} / B_{\text {eff }}$ of the boost factor is also displayed by the short-dashed curve. In the limit where the clump boost factor $B_{\mathrm{c}}$ is large - a value of 100 has been assumed here $-\sigma_{B} / B_{\text {eff }}$ and $\sigma_{r} /\left\langle\phi_{r}\right\rangle$ are approximately equal.

is increased. The relative variance $\sigma_{B} / B_{\text {eff }}$ of the flux enhancement $B \equiv \phi / \phi_{\mathrm{s}}$ is also presented in Fig. 2 . In the limit where the individual clump boost factor $B_{\mathrm{c}}$ is large - we have selected here a value of $B_{\mathrm{c}}=100$ - the random component $\phi_{r}$ of the positron flux dominates over its smooth counterpart $(1-f)^{2} \phi_{\mathrm{s}}$ so that

$\frac{\sigma_{B}}{B_{\mathrm{eff}}}=\frac{\sigma_{r} / \phi_{\mathrm{s}}}{(1-f)^{2}+\left\langle\phi_{r}\right\rangle / \phi_{\mathrm{s}}} \simeq \frac{\sigma_{r}}{\left\langle\phi_{r}\right\rangle}$

That is why the solid lines and short-dashed curves of Fig. 2 are similar. In Fig. 3, the black central curve features the effective boost factor $B_{\text {eff }}$ of a NFW halo and corresponds to the case $B_{\mathrm{c}}=100$ of the panel $\mathrm{b}$ of Fig. 1 from which it has been extracted. The 1- $\sigma$ range of its fluctuations extends from $B_{\text {min }}=B_{\text {eff }}-\sigma_{B}$ up to $B_{\max }=B_{\text {eff }}+\sigma_{B}$. At fixed clump mass, that range opens up as $E$ approaches the injected energy $E_{\mathrm{S}}=100 \mathrm{GeV}$. The fluctuations in the positron signal increase significantly just below the positron line. The boost variance $\sigma_{B}$ is also proportional to $1 / \sqrt{N_{\mathrm{c}}} \propto \sqrt{M_{\mathrm{c}}}$. That is why the fluctuation band broadens as the clump mass is increased from $10^{4}$ to $10^{6} M_{\odot}$.

\subsection{The flux distribution $\mathcal{P}(\varphi)$ of a single clump}

The positron flux at energy $E \leq E_{\mathrm{S}}$ that a single clump located at position $\boldsymbol{x}$ generates at the Earth implies the propagator discussed in Sect. 3

$\varphi(x)=\mathcal{S} \frac{B_{\mathrm{c}} M_{\mathrm{c}}}{\rho_{0}} G_{\mathrm{e}^{+}}\left(x_{\odot}, E \leftarrow \boldsymbol{x}, E_{\mathrm{S}}\right)$. 


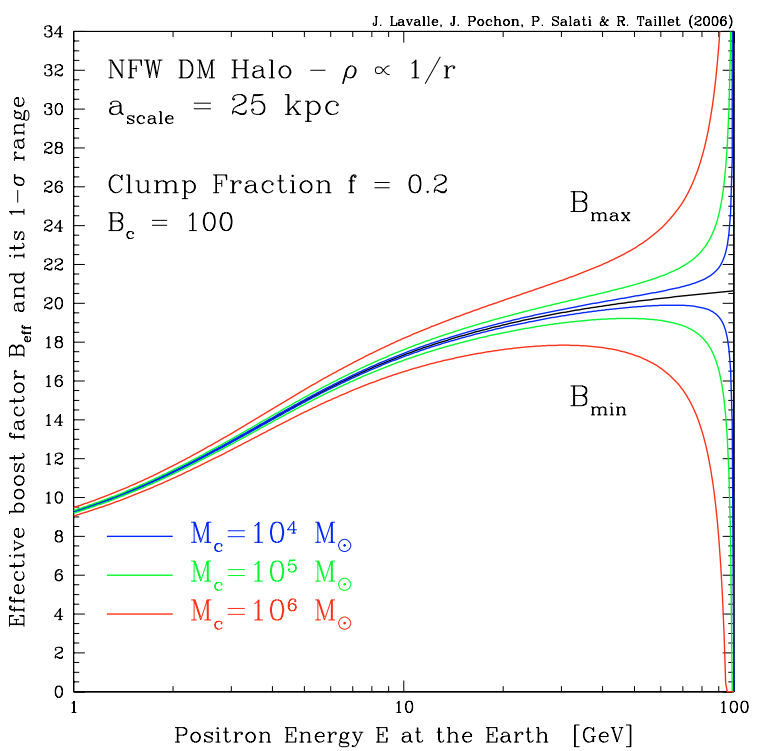

Fig. 3. The effective boost factor $B_{\text {eff }}$ - black line - is plotted as a function of the positron energy $E$ for an injected energy $E_{\mathrm{S}}=100 \mathrm{GeV}$. The 1- $\sigma$ range of its fluctuations extends from $B_{\min }=B_{\text {eff }}-\sigma_{B}$ up to $B_{\max }=B_{\text {eff }}+\sigma_{B}$. At fixed clump mass, that range opens up as $E$ approaches the injected energy $E_{\mathrm{S}}=100 \mathrm{GeV}$. It also widens significantly at fixed positron energy $E$ when the number of clumps is decreased.

and may be expressed with the reduced Green function $\tilde{G}$ as

$\varphi(x)=\mathcal{S} \frac{B_{\mathrm{c}} M_{\mathrm{c}}}{\rho_{0}} \frac{\tau_{E}}{E_{0} \epsilon^{2}} \tilde{G}\left(\boldsymbol{x}_{\odot}, \tilde{t} \leftarrow \boldsymbol{x}, \tilde{t}_{\mathrm{S}}\right)$.

When the substructure is very close to the Earth, the flux $\varphi$ reaches a maximal value $\varphi_{\max }$ that depends both on the clump properties through the effective volume $B_{\mathrm{c}} M_{\mathrm{c}} / \rho_{0}$ and on the specific features assumed for the DM particle through the factor $\mathcal{S}$. Without any loss of generality, we can significantly simplify the discussion by considering the ratio

$\Phi(\boldsymbol{x})=\frac{\varphi(\boldsymbol{x})}{\varphi_{\max }}=\frac{\tilde{G}(\boldsymbol{x})}{\tilde{G}_{\max }}$,

instead of the flux $\varphi$ itself. We therefore would like to derive the density of probability $\mathcal{P}(\Phi)$ associated with the reduced flux $\Phi$ as it varies from 0 to 1 .

In Fig. 4, that distribution is presented for three typical values of the positron energy $E$ at the Earth. The energy $E_{\mathrm{S}}$ of the positron line has been set equal to $100 \mathrm{GeV}$ and a NFW DM halo has been assumed. The solid curves correspond to the fully numerical calculation of $\mathcal{P}(\Phi)$ based on relation (57). The domain $\mathcal{D}_{\mathrm{H}}$ over which the probability is normalized to unity is the Milky Way DM halo up to a radius of $20 \mathrm{kpc}$. That domain encompasses the diffusive halo outside of which the cosmic ray density vanishes. Most of the probability is therefore contained in the low flux region and the density $\mathcal{P}(\Phi)$ diverges at $\Phi=0$. As the energy $E$ increases towards $E_{\mathrm{S}}$, the region of the diffusive halo that is probed by the positron propagator shrinks. That region corresponds to large values of the positron flux $\Phi$. As its volume decreases when $E$ approaches $E_{\mathrm{S}}$, fewer clumps are involved in the signal and the corresponding probability decreases. Notice in Fig. 4 how the probability density $\mathcal{P}(\Phi)$ drops when $E$ is increased from $1 \mathrm{GeV}$ to $50 \mathrm{GeV}$. The lower curve is reproduced in Fig. 5 together with the distributions $\Phi \mathcal{P}(\Phi)$ and

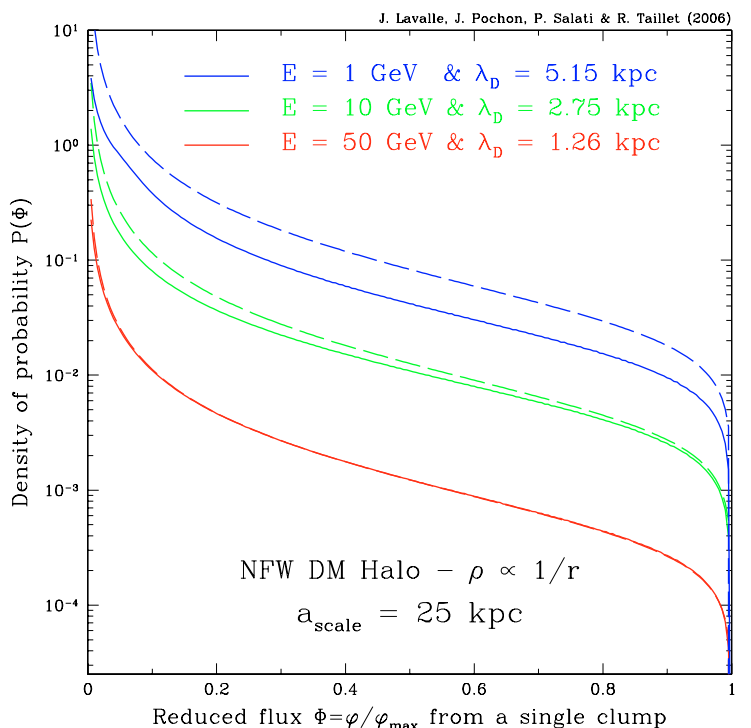

Fig. 4. The density of probability $\mathcal{P}(\Phi)$ as a function of the reduced flux $\Phi=\varphi / \varphi_{\max }$ that a single clump generates. A NFW halo has been assumed with a scale radius of $25 \mathrm{kpc}$. The domain $\mathcal{D}_{\mathrm{H}}$ over which the probability is normalized to unity is the Milky Way DM halo up to a radius of $20 \mathrm{kpc}$. The injection energy is $E_{\mathrm{S}}=100 \mathrm{GeV}$. The smaller the positron energy $E$ at the Earth, the larger the probability density for a non-vanishing flux. The fully numerical calculations - solid curves are compared to the infinite 3D approximation (71) that corresponds to the long-dashed lines.

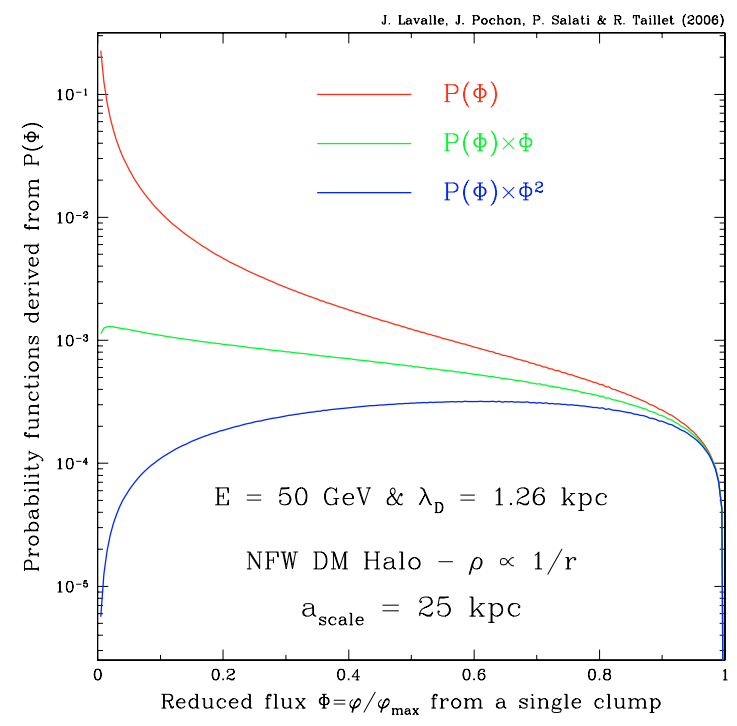

Fig. 5. The density of probability $\mathcal{P}(\Phi), \Phi \mathcal{P}(\Phi)$ and $\Phi^{2} \mathcal{P}(\Phi)$ are featured as a function of the reduced flux $\Phi=\varphi / \varphi_{\max }$ for a positron energy at the Earth of $50 \mathrm{GeV}$.

$\Phi^{2} \mathcal{P}(\Phi)$ whose integrals from $\Phi=0$ up to $\Phi=1$ are respectively related to $\langle\varphi\rangle$ and $\left\langle\varphi^{2}\right\rangle$.

When the positron energy $E$ is close to the energy $E_{\mathrm{S}}$, the pseudo-time difference $\tilde{\tau}=\tilde{t}-\tilde{t}_{\mathrm{S}}$ is so small that the diffusion is no longer sensitive to the vertical boundaries at $z=-L$ and $z=+L$. The Green function $\tilde{G}$ can be safely approximated by the Gaussian function (see Eq. (32))

$\tilde{G}\left(\boldsymbol{x}_{\odot}, \tilde{t} \leftarrow \boldsymbol{x}, \tilde{t}_{\mathrm{S}}\right)=\left\{4 \pi K_{0} \tilde{\tau}\right\}^{-3 / 2} \exp \left\{-\frac{r^{2}}{4 K_{0} \tilde{\tau}}\right\}$. 


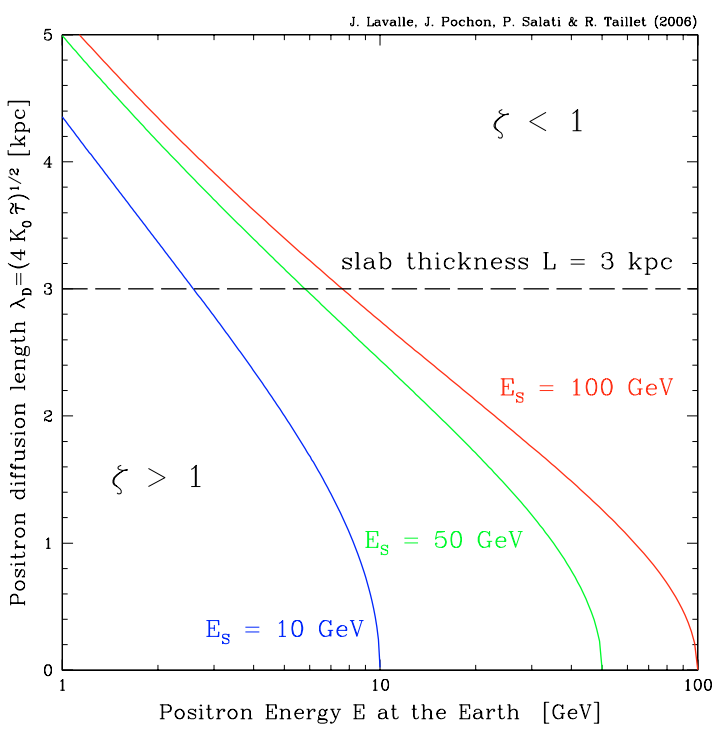

Fig. 6. The positron diffusion length $\lambda_{\mathrm{D}}$ decreases as the energy $E$ at the Earth approaches the energy $E_{\mathrm{S}}$ of the line. The long-dashed horizontal line corresponds to a diffusion length $\lambda_{\mathrm{D}}$ equal to the thickness $L$ of the diffusion layers. Below that limit, positron propagation is not sensitive to the vertical boundaries and the infinite $3 \mathrm{D}$ approximation is valid. This regime corresponds to large values of the parameter $\zeta$ - see the definition (42).

This regime corresponds to large values of the parameter $\zeta-$ defined in relation (42) - or alternatively to small values of the positron diffusion length $\lambda_{\mathrm{D}} \equiv \sqrt{4 K_{0} \tilde{\tau}}$. The latter is featured in Fig. 6 as a function of $E$ for three different values of the energy at source. In the case where $E_{\mathrm{S}}=100 \mathrm{GeV}$, the diffusion length $\lambda_{\mathrm{D}}$ exceeds the thickness $L$ below an energy of $\sim 8 \mathrm{GeV}$. Above that limit, positron propagation is not affected by the vertical boundaries and the infinite 3D approximation (69) applies with a reduced flux $\Phi$ that only depends on the distance $r$ of the clump to the Earth

$\Phi=\exp \left(-r^{2} / \lambda_{\mathrm{D}}^{2}\right)$.

An analytic density of probability may be derived in that case with

$\mathcal{P}(\Phi)=2 \pi \lambda_{\mathrm{D}}^{3} \frac{\rho_{\mathrm{s}}(\odot)}{M_{\mathrm{H}}} \frac{\sqrt{-\ln \Phi}}{\Phi}$.

That relation corresponds to the long-dashed curves of Fig. 4 where a value of $M_{\mathrm{H}}=1.357 \times 10^{11} M_{\odot}$ has been found for the mass contained in the inner $20 \mathrm{kpc}$ of the Milky Way DM halo. When the positron diffusion length $\lambda_{\mathrm{D}}$ is smaller than the slab thickness $L$, relation (71) is an excellent approximation to the density of probability $\mathcal{P}(\Phi)$. As an illustration, we find a value of $\lambda_{\mathrm{D}}=1.26 \mathrm{kpc}$ well below $L=3 \mathrm{kpc}$ when the positron energy $E$ is equal to $50 \mathrm{GeV}$. This explains why the solid and long-dashed red lines of Fig. 4 are so well superimposed. As $E$ decreases, the diffusion length $\lambda_{\mathrm{D}}$ becomes larger with respect to $L$ and the infinite $3 \mathrm{D}$ propagator (69) tends to overestimate the region from which the signal originates as well as the corresponding probability density $\mathcal{P}(\Phi)$. Notice how the long-dashed approximation lines are shifted upwards with respect to the solid true numerical curves in Fig. 4. As $E$ decreases, the approximation (71) worsens and the disagreement with the correct result becomes more pronounced.

\section{The hard-sphere approximation}

In the limit where the infinite $3 \mathrm{D}$ approximation applies - actually for a large range of values of the positron energy $E$ at the Earth - we can further simplify the propagator $G_{\mathrm{e}^{+}}$and substitute the step function

$\tilde{G}\left(\boldsymbol{x}_{\odot}, \tilde{t} \leftarrow \boldsymbol{x}, \tilde{t}_{\mathrm{S}}\right)=\frac{\theta\left(r_{\mathrm{S}}-r\right)}{V_{\mathrm{S}}}$

for the Gaussian form (69). The distance between the clump and the Earth is $r \equiv\left|\boldsymbol{x}-\boldsymbol{x}_{\odot}\right|$. According to this hard-sphere approximation, the Green function $\tilde{G}$ reaches the constant value $1 / V_{\mathrm{S}}$ inside the sphere $\mathcal{D}_{\mathrm{S}}$ of radius $r_{\mathrm{S}}$ and volume $V_{\mathrm{S}}$ - whose center coincides with the Earth - and vanishes elsewhere. Both expressions (69) and (72) are normalized to unity. The integral over the full 3D space of the square of those Green functions should also be the same. This condition translates into

$\frac{1}{V_{\mathrm{S}}}=\int \tilde{G}^{2} \mathrm{~d}^{3} \boldsymbol{x}$

and leads to the volume

$V_{\mathrm{S}}=\left(\sqrt{2 \pi} \lambda_{\mathrm{D}}\right)^{3}$.

In spite of its crudeness, the hard-sphere approximation turns out to be quite powerful and is an excellent tool to understand the salient features of the statistical properties of the clump distribution and of its flux. The associated density of probability has little to do with the curves of Fig. 4 or with relation (71). It is actually a bimodal distribution with

$\mathcal{P}(\Phi)=p \delta(\Phi-1)+(1-p) \delta(\Phi)$.

The reduced flux $\Phi$ takes the value of 1 inside the sphere $\mathcal{D}_{\mathrm{S}}$ and 0 outside. The probability $p$ that a clump lies inside the domain $\mathcal{D}_{\mathrm{S}}$ - from which it may yield a signal at the Earth - is the ratio $M_{\mathrm{S}} / M_{\mathrm{H}}$ of the DM mass $M_{\mathrm{S}}$ confined in that sphere with respect to the DM mass $M_{\mathrm{H}}$ contained in the entire domain $\mathcal{D}_{\mathrm{H}}$. In the limit where $\lambda_{\mathrm{D}} \propto r_{\mathrm{S}}$ is small, the DM distribution is homogeneous inside the sphere $\mathcal{D}_{\mathrm{S}}-$ with constant density $\rho_{\mathrm{S}}(\odot)-$ and the probability $p$ may be expressed as the ratio

$p=\frac{M_{\mathrm{S}}}{M_{\mathrm{H}}}=\frac{V_{\mathrm{S}} \rho_{\mathrm{S}}(\odot)}{M_{\mathrm{H}}}$.

For an injected energy $E_{\mathrm{S}}=100 \mathrm{GeV}$ and a positron energy at the Earth $E=50 \mathrm{GeV}$, we find a probability $p \sim 2 \times 10^{-3}$ when the statistical domain $\mathcal{D}_{\mathrm{H}}$ is chosen to be the above-mentioned NFW halo extending up to $20 \mathrm{kpc}$ from the center of the Milky Way.

Because $p$ is vanishingly small and the number of clumps $N_{\mathrm{H}}$ inside the domain $\mathcal{D}_{\mathrm{H}}$ exceedingly large, the limit of Poisson statistics is reached. The probability to find $n$ clumps inside the sphere $\mathcal{D}_{\mathrm{S}}$ is therefore given by

$P(n)=\frac{N_{\mathrm{S}}{ }^{n}}{n !} \exp \left(-N_{\mathrm{S}}\right)$,

where $N_{\mathrm{S}} \equiv p N_{\mathrm{H}}$ is the average number of clumps that contribute to the signal

$\langle n\rangle=N_{\mathrm{S}}=\frac{V_{\mathrm{S}} f \rho_{\mathrm{S}}(\odot)}{M_{\mathrm{c}}}$.

Departures from the statistical law (77) in the case of a realistic positron propagator will be discussed in Sect. 6.1 when the number $N_{\mathrm{S}}$ of the clumps involved in the flux at the Earth is large 
whereas the opposite regime will be addressed in Sect. 6.2. The Poisson distribution (77) is associated with the variance

$\sigma_{n}^{2}=\left\langle n^{2}\right\rangle-\langle n\rangle^{2}=N_{\mathrm{S}}$

In the hard-sphere approximation, the random part $\phi_{r}$ of the positron flux at the Earth - the contribution that the entire constellation of substructures generates - is proportional to the number $n$ of clumps lying inside the sphere $\mathcal{D}_{\mathrm{S}}$. We therefore anticipate that the relative variance $\sigma_{r} /\left\langle\phi_{r}\right\rangle$ should be equal to the relative variance $\sigma_{n} /\langle n\rangle$ of the Poisson law (77). In the limit where $\lambda_{\mathrm{D}}$ is small with respect to $L$ - and where the hard-sphere approximation becomes valid - the integrals $\mathcal{J}_{1}$ and $\mathcal{I}_{1}$ simplify. If the mass density of reference $\rho_{0}$ is set equal to its solar neighbourhood value $\rho_{\mathrm{S}}(\odot)$, the ratio $\mathcal{J}_{1} / \mathcal{I}_{1}^{2}$ is $1 / V_{\mathrm{S}}$ so that the exact relation (64) simplifies into

$\frac{\sigma_{r}^{2}}{\left\langle\phi_{r}\right\rangle^{2}}=\frac{M_{\mathrm{c}}}{f \rho_{0}} \frac{\mathcal{J}_{1}}{\mathcal{I}_{1}^{2}}=\frac{M_{\mathrm{c}}}{V_{\mathrm{S}} f \rho_{\mathrm{S}}(\odot)}=\frac{1}{N_{\mathrm{S}}}$.

We have therefore shown that in the hard-sphere regime, the variance $\sigma_{r}$ of the random flux $\phi_{r}$ is indeed given by the variance $\sigma_{n}$ that characterizes the Poisson statistics (77). In Fig. 2, the relative variance $\sigma_{r} /\left\langle\phi_{r}\right\rangle$ - solid curves - and its hard-sphere approximation $1 / \sqrt{N_{\mathrm{S}}}$ - long-dashed lines - are presented together for comparison. Above a positron energy at the Earth of $40 \mathrm{GeV}$, the correct calculation and its hard-sphere limit differ by less than $\sim 5 \times 10^{-3}$. The agreement is remarkable. The diffusion length does not exceed $\sim 1.5 \mathrm{kpc}$ in that case and the hard-sphere approximation successfully describes the statistical properties of the random positron flux $\phi_{r}$. The relative variance $\sigma_{B} / B_{\text {eff }}$ of the boost factor is also well reproduced by the hard-sphere approximation $1 / \sqrt{N_{\mathrm{S}}}$ and both the short-dashed and long-dashed curves are barely distinguishable from each other at high positron energy $E$.

\section{A Monte-Carlo approach to the cosmic ray flux fluctuations}

\subsection{The large $N_{S}$ limit and the central limit theorem}

When the average number $N_{\mathrm{S}}$ of clumps that are involved in the signal is large, the Poisson statistics (77) becomes the Gaussian distribution

$P(\delta)=\frac{1}{\sqrt{2 \pi N_{\mathrm{S}}}} \exp \left(-\delta^{2} / 2 N_{\mathrm{S}}\right)$,

where $\delta \equiv n-N_{\mathrm{S}}$ denotes the departure of the number $n$ of substructures inside the sphere $\mathcal{D}_{\mathrm{S}}$ from its average value $N_{\mathrm{S}}$. The associated variance is $\sigma_{n}=\sqrt{N_{\mathrm{S}}}$. We therefore anticipate that the flux $\phi_{r}$ will also be randomly distributed according to a Gaussian law with mean value $\left\langle\phi_{r}\right\rangle$ and variance $\sigma_{r}$.

In order to determine the distribution of probability $\mathcal{P}\left(\phi_{r}\right)$ that drives the random flux $\phi_{r}-$ generated by the entire constellation of the clumps lying inside the reservoir $\mathcal{D}_{\mathrm{H}}-$ we should compute the product of convolution of the $N_{\mathrm{H}}$ distributions of probability $\mathcal{P}(\varphi)$ associated each with the flux $\varphi$ of a single substructure - or alternatively with its reduced flux $\Phi$ as was discussed in Sect. 4.2. In the large $N_{\mathrm{S}}$ regime, the central limit theorem may be applied. This theorem states that the product of convolution is a Gaussian distribution with mean value $\left\langle\phi_{r}\right\rangle \equiv N_{\mathrm{H}}\langle\varphi\rangle$ and variance $\sigma_{r}^{2} \equiv N_{\mathrm{H}}\left\{\left\langle\varphi^{2}\right\rangle-\langle\varphi\rangle^{2}\right\}$. These are
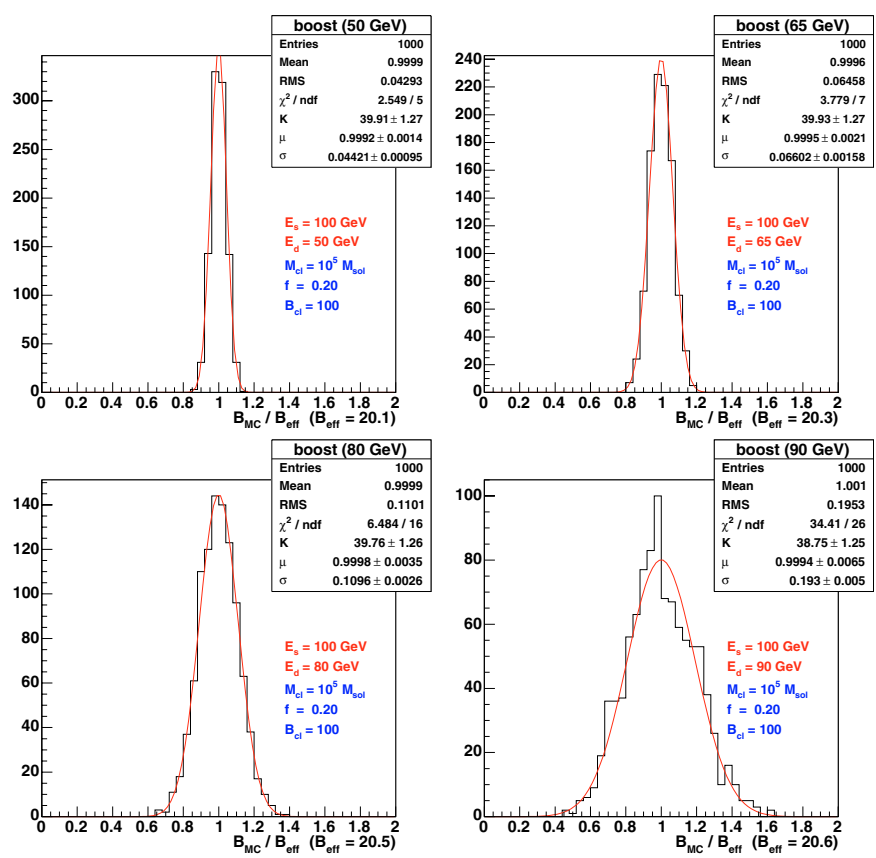

Fig. 7. One thousand different realizations of the distribution of DM substructures inside the galactic halo has been generated by MonteCarlo simulation. The injected energy is $E_{\mathrm{S}}=100 \mathrm{GeV}$. A NFW profile has been assumed with typical scale $25 \mathrm{kpc}$. A mass fraction $f=0.2$ is in the form of clumps with mass $10^{5} M_{\odot}$. Each histogram corresponds to a specific positron energy $E$ at the Earth. The number of realizations each involving 271, 488 clumps - is plotted as a function of the reduced boost $\eta=B / B_{\text {eff }}$. Up to an overall factor of a thousand - that corresponds to the number of Monte-Carlo realizations - each panel features a numerical estimate of the probability density $\mathcal{P}(\eta)$.

expressions (53) and (54) which have been established and numerically computed in Sect. 4.1. Therefore, the probability of obtaining a flux $\phi_{r}$ at the Earth is

$\mathcal{P}\left\{\phi_{r}=\sum_{i} \varphi_{i}\right\}=\frac{1}{\sqrt{2 \pi \sigma_{r}^{2}}} \exp \left\{-\frac{\left(\phi_{r}-\left\langle\phi_{r}\right\rangle\right)^{2}}{2 \sigma_{r}^{2}}\right\}$.

The probability that the total positron flux $\phi$ at the Earth is enhanced by a factor of $B$ with respect to a completely smooth DM distribution is

$\mathcal{P}\left\{B \equiv \phi / \phi_{\mathrm{s}}\right\}=\frac{1}{\sqrt{2 \pi \sigma_{B}^{2}}} \exp \left\{-\frac{\left(B-B_{\mathrm{eff}}\right)^{2}}{2 \sigma_{B}^{2}}\right\}$,

where the variance $\sigma_{B}$ is given by relation (52). Finally the reduced boost $\eta \equiv B / B_{\text {eff }}$ follows the same Gaussian law

$\mathcal{P}\left\{\eta \equiv B / B_{\mathrm{eff}}\right\}=\frac{1}{\sqrt{2 \pi \sigma_{\eta}^{2}}} \exp \left\{-\frac{(\eta-1)^{2}}{2 \sigma_{\eta}^{2}}\right\}$,

with an average value of $\langle\eta\rangle=1$ and a variance $\sigma_{\eta}=\sigma_{B} / B_{\text {eff }}$ not too different from $\sigma_{r} /\left\langle\phi_{r}\right\rangle$ as shown in formula (65).

To check our theoretical predictions, we ran a Monte-Carlo simulation of the distribution of DM substructures in the Milky Way halo. A thousand different realizations were generated at random assuming a NFW DM galactic halo with a fraction $f=0.2$ in the form of $10^{5} M_{\odot}$ clumps. In Fig. 7 , the number of realizations is plotted as a function of the reduced boost $\eta$ for 4 values of the positron energy at the Earth. These distributions 
Table 1. For each value of the positron energy $E$ at the Earth that has been considered in the plots of Fig. 7, we have computed the corresponding effective boost $B_{\text {eff }}$ as well as the variance $\sigma_{\eta}$ associated with the reduced boost $\eta=B / B_{\text {eff }}$. That variance has been derived from relation (65) and is in excellent agreement with the rms value of the MonteCarlo simulation. The average number $N_{\mathrm{S}}$ of substructures inside the sphere $\mathcal{D}_{\mathrm{S}}$ is indicated.

\begin{tabular}{cccc}
\hline \hline$E$ & $B_{\text {eff }}$ & $\sigma_{\eta}=\sigma_{B} / B_{\text {eff }}$ & $N_{\mathrm{S}}$ \\
\hline 50 & 20.09 & 0.04338 & 498.0 \\
65 & 20.32 & 0.06472 & 223.5 \\
80 & 20.48 & 0.10966 & 78.0 \\
90 & 20.57 & 0.19680 & 24.2 \\
\hline
\end{tabular}

are the Monte-Carlo counterparts of the Gaussian law (84) with a mean value of $\eta$ very close to 1 . In each panel, the rms value of the histogram is equal - within a few percent - to the variance $\sigma_{\eta}=\sigma_{B} / B_{\text {eff }}$ which we have derived from expression (65) and listed in Table 1 for comparison with the results of Fig. 7. For completeness, each histogram has been independently fitted by the Gaussian distribution

$\mathcal{G}(\eta, \mu, \sigma)=\frac{K}{\sqrt{2 \pi \sigma^{2}}} \exp \left\{-\frac{(\eta-\mu)^{2}}{2 \sigma^{2}}\right\}$.

The amplitude $K$, mean value $\mu$ and variance $\sigma$ are displayed in each panel and the corresponding fitted Gaussian is shown by the red curve. The width of each bin is $\Delta \eta=0.04$ and since we have generated $10^{3}$ Monte-Carlo realizations, we expect a value of $K=0.04 \times 10^{3}=40$ for the amplitude. This is what we obtain. The mean value $\mu$ of the Gaussian is 1 whereas its variance $\sigma$ is very close to the Monte-Carlo rms value and to $\sigma_{\eta}$ - see Table 1 . Because the clumps that are involved in the positron signal at the Earth are numerous - the average number $N_{\mathrm{S}}$ is still larger than $\sim 20$ even at the highest energy $E=90 \mathrm{GeV}$ - the central limit theorem applies and the Gaussian distribution (84) is an excellent description of the statistical fluctuations of the positron flux. The question is now to understand how the distribution of probability $\mathcal{P}(\eta)$ is modified in the limit where $N_{\mathrm{S}}$ becomes smaller than 1. This is illustrated in Fig. 8.

\subsection{The small $N_{S}$ regime}

When the diffusion range of positrons is small (for energies close to the emission energy), the individual probability distribution $\mathcal{P}_{1}(\Phi) \equiv \mathcal{P}(\Phi)$ (where $\Phi \equiv \varphi / \varphi_{\max }$ ) is strongly peaked at $\Phi_{\min } \sim 0$. As a result, the probability distribution for the total flux $\Phi_{\text {tot }}$ generated by the $N \equiv N_{\mathrm{H}}$ clumps of domain $\mathcal{D}_{\mathrm{H}}$ can be approximated by

$\mathcal{P}_{N}\left(\Phi_{\text {tot }}\right)=N \mathcal{P}_{1}\left(\Phi_{\text {tot }}\right)$

for $0<\Phi_{\text {tot }}<1$ and in the regime where $\left\langle\Phi_{\text {tot }}\right\rangle$ is vanishingly small. The proof is straightforward. The probability $\mathcal{P}_{N}$ is given by

$\mathcal{P}_{N}\left(\Phi_{\mathrm{tot}}\right)=\int_{0}^{1} \mathcal{P}_{1}(\Phi) \mathcal{P}_{N-1}\left(\Phi_{\mathrm{tot}}-\Phi\right) \mathrm{d} \Phi$

When $\mathcal{P}_{N-1}\left(\Phi_{\text {tot }}\right)$ behaves qualitatively like $\mathcal{P}_{1}(\Phi)$ and is strongly peaked at a value close to 0 , two regions dominate the contribution to the integral when $\Phi_{\text {tot }}<1$, namely $\Phi$ close to 0 (where $\mathcal{P}_{1}(\Phi)$ is large) and $\Phi$ close to $\Phi_{\text {tot }}$ (where $\mathcal{P}_{N-1}\left(\Phi_{\text {tot }}-\Phi\right)$ is large), so that

$\mathcal{P}_{N}\left(\Phi_{\text {tot }}\right) \approx \mathcal{P}_{N-1}\left(\Phi_{\text {tot }}\right)+\mathcal{P}_{1}\left(\Phi_{\text {tot }}\right)$
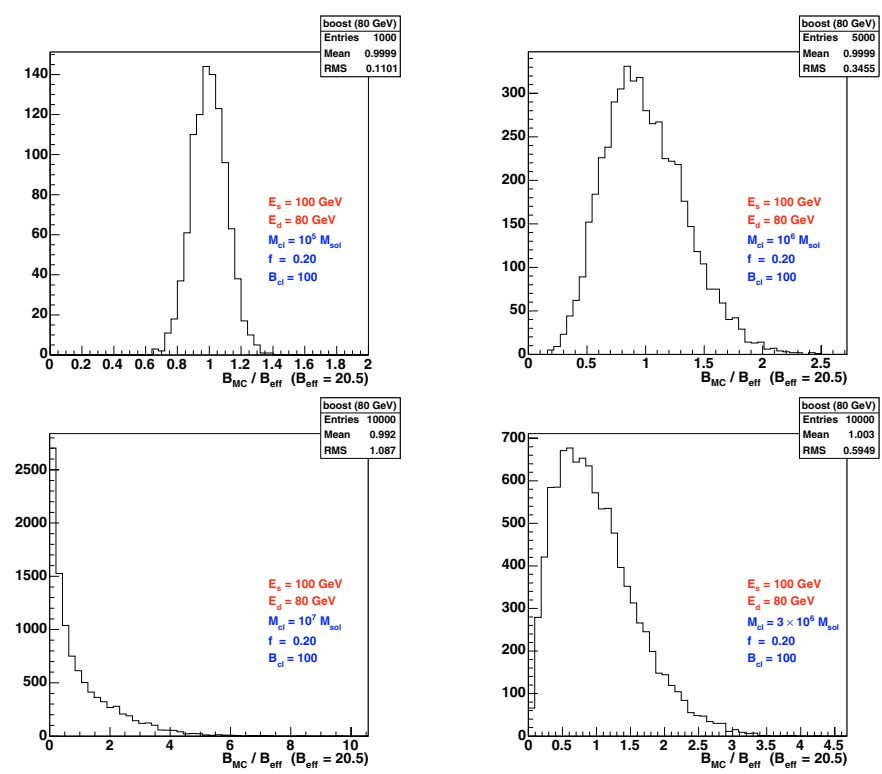

Fig. 8. Distribution of $B_{\mathrm{MC}} / B_{\text {eff }}$ for an energy $E=80 \mathrm{GeV}$, given the source energy $E_{\mathrm{S}}=100 \mathrm{GeV}$, with a mass $M_{\mathrm{c}}$ of individual clumps equal to $10^{5} M_{\odot}, 10^{6} M_{\odot}, 3 \times 10^{6} M_{\odot}$ and $10^{7} M_{\odot}$. When $M_{\mathrm{c}}$ is small, the clumps are numerous enough for the central limit theorem to apply. The mass fraction in clumps is set to the value $f=0.2$. The resulting distribution is a Gaussian, as described in the text. On the other hand, when $M_{\mathrm{c}}$ is large, the probability that several clumps contribute to the observed signal is small and the observed distribution for $B_{\mathrm{MC}} / B_{\text {eff }}$ reflects the one clump distribution $P_{1}(\Phi)$.

which proves the property (86). This is illustrated in Fig. 9 where the distributions $\mathcal{P}_{1}, \mathcal{P}_{2} / 2$ and $\mathcal{P}_{3} / 3$ are featured as a function of the total positron flux expressed in units of the maximal value $\varphi_{\max }$ which a single clump can generate at the Earth. The self convolution of $P_{1}(\Phi)$ has been carried out numerically to yield $\mathcal{P}_{2}$ and $\mathcal{P}_{3}$, assuming relation (71). The size of the statistical domain $\mathcal{D}_{\mathrm{H}}$ has been fixed by setting a low flux cut-off of $\Phi_{\min }=0.001$. As is clear in Fig. 9, the three distributions are similar in the range where $\Phi$ is smaller than 1 . This is so because the probability densities are peaked at $\Phi=0$. Should we have chosen a smaller domain $\mathcal{D}_{\mathrm{H}}$ and hence a larger value for the cut-off $\Phi_{\min }$, the distributions would have been less saturated by their low-flux behaviour and relation (86) would not have applied. In Fig. 10, $10^{5}$ realizations of a clumpy DM halo have been simulated with a substructure mass of $10^{7} M_{\odot}$. On the horizontal axis, the histogram features the boost ratio $\eta \equiv B / B_{\text {eff }}$ which is proportional to $\Phi_{\text {tot }}$. The resemblance with the analytical distributions of Fig. 9 is striking. The red curve that is superimposed on the Monte-Carlo results of Fig. 10 corresponds to the product

$N_{\mathrm{H}} \mathcal{P}_{1}(\varphi) \equiv \frac{f}{M_{\mathrm{c}}} \int_{\mathcal{D}_{\varphi}} \rho_{\mathrm{s}}(\boldsymbol{x}) \mathrm{d}^{3} \boldsymbol{x}$

with the same values of $f$ and $M_{\mathrm{c}}$ as in the simulation. On a large portion of the range extending from $\sim 0$ up to $B \sim 11 B_{\text {eff }}$ - therefore for a total flux smaller than $\varphi_{\max }-$ relation (86) is a quite good approximation. This regime corresponds to the situation where a single clump happens to contribute significantly more than the others and is the framework of the Cumberbatch \& Silk (2006) work. Most of the realizations of Fig. 10 correspond to small values of the flux ratio $\Phi_{\text {tot }}$. The number of clumps effectively implied in the signal is on average very small $\left(N_{\mathrm{S}} \ll 1\right)$. 


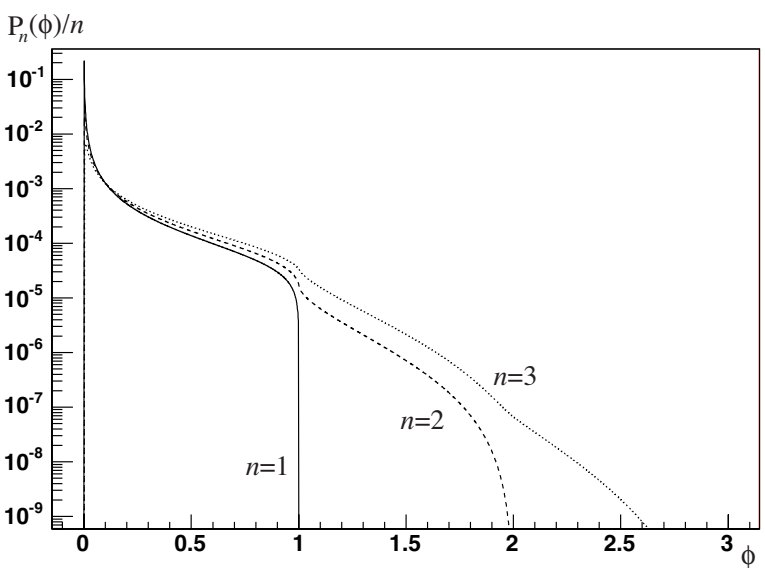

Fig. 9. Probability distribution $\mathcal{P}_{n}(\Phi) / n$ for $n=1,2$ and 3 , obtained by consecutive convolutions of $\mathcal{P}_{1}(\Phi)$.

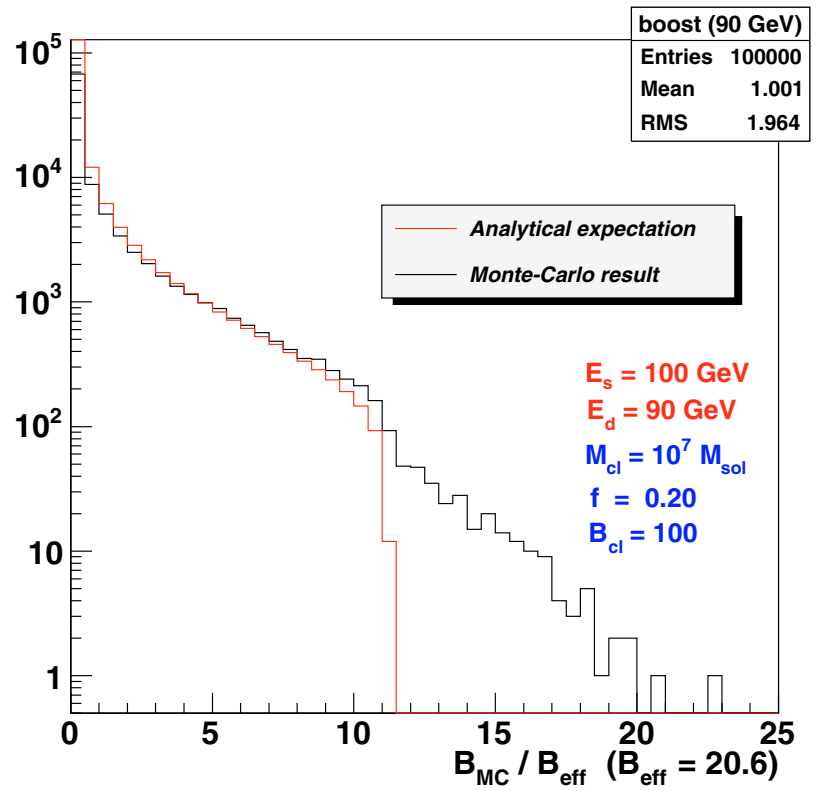

Fig. 10. Probability distribution of $B / B_{\text {eff }}$ for an assembly of clumps and the corresponding distribution for one clump, multiplied by $N$ (see text). The curves are similar in their common range, as is expected from an analytical calculation of $\mathcal{P}_{N}(\Phi)$ (see Fig. 9).

\section{A practical example: HEAT excess}

The putative positron excess reported by the HEAT experiment around $\sim 10 \mathrm{GeV}$ is suggestive of an exotic mechanism such as for example the annihilation of wimps potentially concealed inside the halo of the Milky Way.

The present study sketches a well defined frame that can be used to make predictions with respect to available DM candidates and experimental data. HEAT measurements (Barwick et al. 1997; Coutu et al. 2001) have been widely exploited in connection to annihilating DM, and we can easily verify how our results translate into phenomenology. For that purpose, we have chosen an illustration based on a DM candidate that was proposed in the so-called warped GUT theoretical scheme (Agashe \& Servant 2004). Within such an extra-dimensional modelling, the conservation of a discrete symmetry called $Z_{3}$-symmetry (which is related to the stabilization of the proton, like the
$R$-parity in supersymmetry), allows the survival of the lightest $Z_{3}$-charged particle, which has the properties of a right-handed neutrino called LZP hereafter. This particle is actually a Dirac fermion, and given that no matter/anti-matter asymmetry is involved in that case, the annihilation rate per volume unit of this species is provided by equation 1 with $\delta=1 / 4$. We thus have considered a fiducial model in which we fixed the LZP mass to $50 \mathrm{GeV}$, and the extra-dimension mass parameter to $m_{K K}=6 \mathrm{TeV}$. The cross-section formulae can be found in Agashe \& Servant (2004), and are mainly defined by the isospin content of any final state. About $10 \%$ of the annihilation product is carried out equally by the three charged lepton/anti-lepton pairs, which can provide a relevant contribution to a sharp component close to the wimp mass in the injected positron spectrum. We then used the Pythia (Sjöstrand et al. 2001) Monte-Carlo program to infer the positron spectrum associated with all decay and fragmentation processes.

To calculate the expected positron fraction, we made a further assumption, that to each positron generated by wimp annihilation and propagated to the Earth, an electron is associated with the same spectral information. The positron fraction is consequently given by the following expression:

$$
f_{\mathrm{e}^{+}}(E)=\frac{\phi_{\mathrm{e}^{+}}(E)+\phi_{\mathrm{e}^{+}, \mathrm{gg}}(E)}{\phi_{\mathrm{e}^{+}}(E)+\phi_{\mathrm{e}^{+}, \mathrm{bg}}(E)+\phi_{\mathrm{e}^{-}}(E)+\phi_{\mathrm{e}^{-}, \mathrm{bg}}(E)}
$$

where $b g$ indicates non-exotic contributions (secondary for positrons, both primary and secondary for electrons). For those components, we used the estimates by Moskalenko \& Strong (1998).

We show in Fig. 11 the results obtained when considering an NFW profile, and $f=20 \%$ of the halo mass within a radius of $20 \mathrm{kpc}$ being clumpy. We will not discuss how the obtained spectrum is compatible with previous work. Instead, we stress here the differences between the naive account of a global and wrong boost factor set by the product $f \times B_{\mathrm{c}}$, and the correct treatment of the problem that we have presented in this paper. To this aim, we used two particular Monte-Carlo simulations with clump masses of $10^{7} M_{\odot}$ and individual boost factor of $B_{\mathrm{c}}=200$. This illustrates the situation where a small number of clumps contributes,

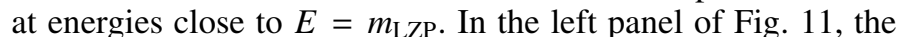
closest clump is found to lie at a distance of $\sim 1 \mathrm{kpc}$ to the Earth, that corresponds to a regime in which $B / B_{\text {eff }}<1$ (cf. Fig. 10). In the right panel, the closest clump has a distance to the Earth of $\sim 0.25 \mathrm{kpc}$, which is a much less probable configuration, with $B / B_{\text {eff }}>5$. The corresponding probability is less than $1 \%$, as shown by Fig. 10. In both panels, the solid blue curves feature the correct treatment of the boost factor, while the green lines correspond to the naive shift by a factor of $f \times B_{\mathrm{c}}$, 40 in this case. Notice the discrepancy at low energy in both panels, that is consequent to the energy dependence of the correct boost factor $B_{\text {eff }}$. In the right panel, the Monte-Carlo result is five times larger than the naively boosted flux close to $M_{\mathrm{LZP}}=50 \mathrm{GeV}$, as a result of the variance affecting this small $N_{\mathrm{S}}$ configuration.

This indicates how carefully predictions should be made when computing the flux enhancement due to clumpiness. We stress that the variance of that boost should also be provided along with the mean values. The spectral distorsions could be sizeable when compared to the experimental error bars of the HEAT results. It is of paramount importance to properly take them into account when studying the discovery potential of the next generation experiments, such as AMS (AMS Collaboration 2006) or PAMELA (PAMELA collaboration 2006). The case of the LZP has been chosen as typical. For particles annihilating 

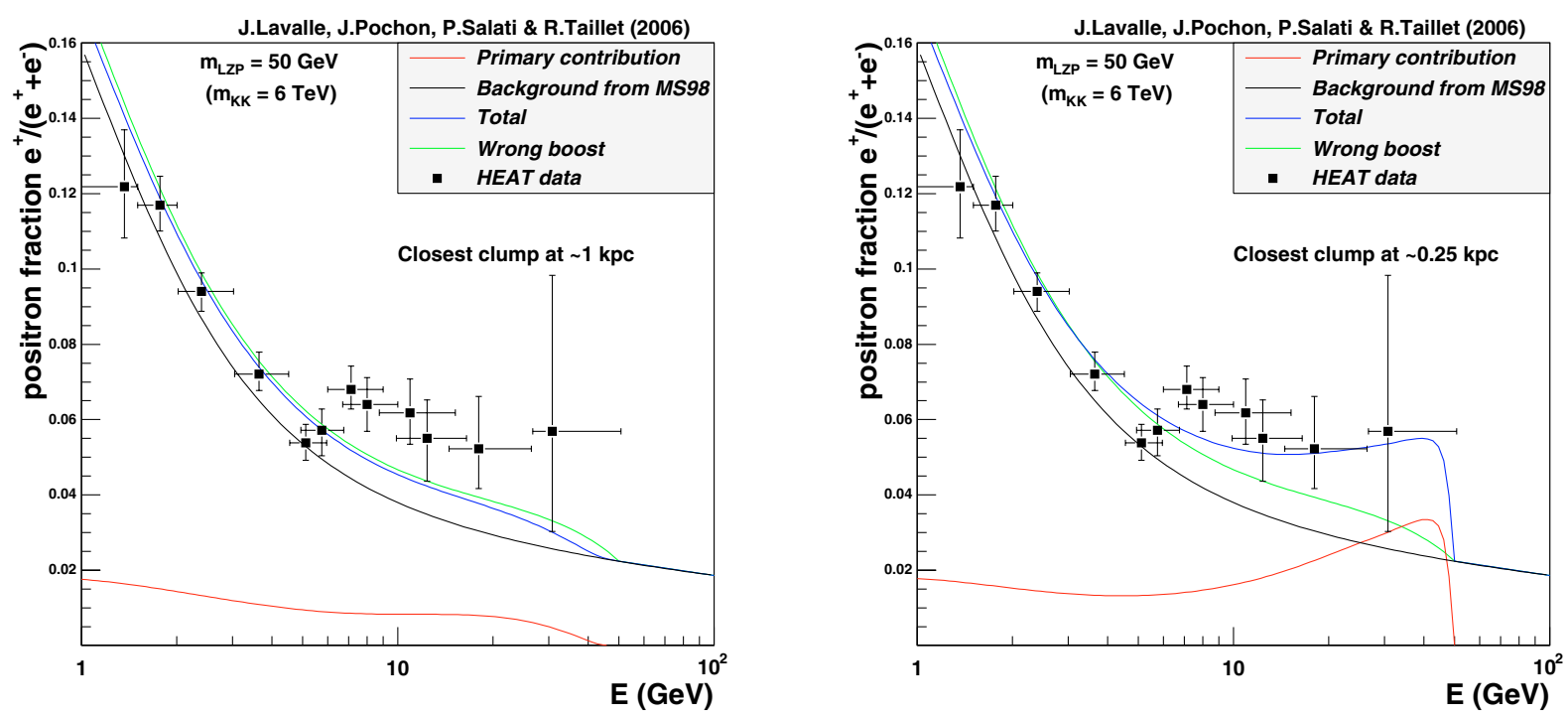

Fig. 11. Expected positron fraction in the frame of LZP DM particles, with $m_{\mathrm{LZP}}=50 \mathrm{GeV}$, superimposed on the HEAT data (Coutu et al. 2001), as computed from two random realizations of a clumpy halo with clump masses of $10^{7} M_{\odot}$. The smooth DM distribution follows an NFW profile with a scale radius of $25 \mathrm{kpc}$, and the mass fraction in clumps within $20 \mathrm{kpc}$ has been set to $f=0.2$. An intrinsic clump boost of $B_{\mathrm{c}}=200$ is considered. Left: the closest clump has a distance to the Earth of $\sim 1 \mathrm{kpc}$. Right: the closest clump has a distance to the Earth of $\sim 0.25 \mathrm{kpc}$, which is less probable than the left-panel case. In both panels, the red and blue curves give respectively the primary and the total (primary + background) contributions from DM annihilation. A smooth halo would not give rise to any excess with respect to the background in that case. For comparison, the green curve illustrates the wrong use of the boost, which is simply a shift of the spectrum by a factor of $f \times B_{\mathrm{c}}, 40$ in that case.

mostly into charged lepton pairs (respectively quark pairs), like the lightest Kaluza-Klein candidate $B^{(1)}$ of universal extradimension theories (neutralinos in mSUGRA) - the effect would be stronger (a bit weaker).

Recently, the presence of local dark matter substructure has been invoked with the specific assumption (Cumberbatch \& Silk 2006) that a single neutralino clump would alone generate the observed distortion in the positron spectrum should it be very close to the Earth - at a distance of $\sim 0.1 \mathrm{pc}$. The contribution of the other and more remote protohalos was assessed to be negligible.

We will not discuss here whether the distortion that that single substructure generates really matches the data or not nor will we be interested in the specific nature of the wimp at stake. Rather, we determine the probability for a nearby clump alone to shine more strongly than the rest of the other protohalos. This situation could indeed arise as suggested by Fig. 11, but its probability is vanishingly small in the Cumberbatch \& Silk (2006) configuration. They have assumed that half of the Milky Way dark matter halo was made of $\sim 10^{15}$ Earth mass clumps as suggested by recent numerical simulations (Diemand et al. 2005). That constellation of neutralino substructures is randomly distributed and contributes on average a positron flux $\left\langle\phi_{r}\right\rangle$ at the Earth. The nearby protohalo yields in addition a signal $\phi_{r}-\left\langle\phi_{r}\right\rangle$ that, according to Cumberbatch \& Silk (2006), overcomes the contribution $\left\langle\phi_{r}\right\rangle$ from the other clumps. The explanation of the HEAT excess in terms of that providential protohalo relies therefore on the assumption that the total positron flux $\phi_{r}$ is larger than twice the average value $\left\langle\phi_{r}\right\rangle$. The probability for such a configuration may be expressed as

$P\left\{\phi_{r} \geq 2\left\langle\phi_{r}\right\rangle\right\}=\int_{2\left\langle\phi_{r}\right\rangle}^{+\infty} \mathcal{P}\left(\phi_{r}\right) \mathrm{d} \phi_{r}$.
The distribution of probability is given by the Maxwell law (82) insofar as the central limit theorem can be applied in that case. The previous relation translates into

$\ln P\left\{\phi_{r} \geq 2\left\langle\phi_{r}\right\rangle\right\}=-a-\ln 2-\frac{1}{2} \ln (\pi a)$,

where the parameter $a$ stands for the ratio $\left\langle\phi_{r}\right\rangle^{2} / 2 \sigma_{r}^{2}$. The relevant statistical quantity which we need to derive is the variance $\sigma_{r}$.

We may readily apply the tools that we have constructed given that we are now dealing with a neutralino that produces a continuous positron spectrum instead of a line. The positron propagator $G_{\mathrm{e}^{+}}\left(\boldsymbol{x}_{\odot}, E \leftarrow \boldsymbol{x}, E_{\mathrm{S}}\right)$ needs to be replaced by the convolution

$$
\begin{aligned}
G^{\prime}(\boldsymbol{x}) & \equiv G_{\mathrm{e}^{+}}^{\prime}\left(\boldsymbol{x}_{\odot}, E \leftarrow \boldsymbol{x}\right) \\
& =\left.\int_{E}^{m_{\chi}} G_{\mathrm{e}^{+}}\left(\boldsymbol{x}_{\odot}, E \leftarrow \boldsymbol{x}, E_{\mathrm{S}}\right) \frac{\mathrm{d} N_{\mathrm{e}^{+}}}{\mathrm{d} E_{\mathrm{e}^{+}}}\right|_{E_{\mathrm{S}}} \mathrm{d} E_{\mathrm{S}},
\end{aligned}
$$

where $\mathrm{d} N_{\mathrm{e}^{+}} / \mathrm{d} E_{\mathrm{e}^{+}}$denotes the positron spectrum at the source. Without loss of generality, we have focused our discussion on a neutralino with mass $m_{\chi}=100 \mathrm{GeV}$ and a positron energy at the Earth of $E=10 \mathrm{GeV}$. Neutralinos have been assumed to annihilate into $b \bar{b}$ pairs. According to the hard-sphere approximation of Sect. 5 , the positrons that are produced at the energy $E_{\mathrm{S}}$ originate from the volume $V_{\mathrm{S}}=\left(\sqrt{2 \pi} \lambda_{\mathrm{D}}\right)^{3}$ surrounding the Earth. In the case that we consider here, the positrons that are detected at the energy $E$ at the Earth have been produced at an energy $E_{\mathrm{S}}$ that spans the entire range from $E$ up to the mass $m_{\chi}$. The volume from which the signal originates on average is the convolution

$V_{\mathrm{S}}^{\prime}=\left.\int_{E}^{m_{\chi}} V_{\mathrm{S}}\left(E, E_{\mathrm{S}}\right) \frac{\mathrm{d} N_{\mathrm{e}^{+}}}{\mathrm{d} E_{\mathrm{e}^{+}}}\right|_{E_{\mathrm{S}}} \mathrm{d} E_{\mathrm{S}}$. 
The number of protohalos that contribute to the positron signal at $10 \mathrm{GeV}$ is inferred to be

$N_{\mathrm{S}} \simeq \frac{V_{\mathrm{S}}^{\prime} f \rho_{\mathrm{s}}(\odot)}{M_{\mathrm{c}}}$,

and the relative variance $\sigma_{r} /\left\langle\phi_{r}\right\rangle$ may be crudely approximated by $1 / \sqrt{N_{\mathrm{S}}}$. With a fraction of clumps of $f=0.5$ and a substructure mass of $M_{\mathrm{c}}=10^{-5} M_{\odot}$, we find a number of protohalos of $N_{\mathrm{S}}=2 \times 10^{13}$ and a relative variance of $\sigma_{r} /\left\langle\phi_{r}\right\rangle \sim 2.22 \times 10^{-7}$. Because of that very large number of clumps, the use of the central limit theorem is plainly justified. The relative variance of the positron signal is vanishingly small and we therefore anticipate that the probability $P\left\{\phi_{r} \geq 2\left\langle\phi_{r}\right\rangle\right\}$ is completely negligible.

The correct calculation of the variance makes use of relation (64) where the integrals $\mathcal{I}_{1}$ and $\mathcal{J}_{1}$ are now computed with the convoluted positron propagator $G^{\prime}(\boldsymbol{x})$. We have derived a value of $\sigma_{r} /\left\langle\phi_{r}\right\rangle \sim 4.13 \times 10^{-7}$ in good agreement with the hardsphere approximation. We therefore reach the conclusion that

$\log _{10} P\left\{\phi_{r} \geq 2\left\langle\phi_{r}\right\rangle\right\}=-1.27 \times 10^{12}$.

With such an exceedingly small value of the probability, the configuration in which a single clump overcomes the signal from the other $2 \times 10^{13}$ substructures is completely unlikely and the hypothesis pursued in Cumberbatch \& Silk (2006) should be abandoned. This example illustrates how the tools presented here may be applied to derive quantitative results.

\section{Discussion and conclusion}

Summary: The enhancement of indirect signals coming from dark matter annihilation in a clumpy halo is usually described by a "boost factor". We have shown that this quantity should be considered as a random variable and we have investigated its statistical properties in the following situations: (i) clumps are distributed like the smooth component; (ii) all the clumps are identical. We showed that the boost factor may strongly depend on the specific realization of the clumpy halo we are living in.

Spatial distribution of clumps: A more realistic model would include the mass profile of these clumps, their own density shape and geometry, as well as a number distribution inspired by hierarchical structure formation studies (which is often found to be close to the smooth distribution, at least at large galactic radii (Berezinsky et al. 2003)). Taking another number distribution would modify the shape of the effective boost factor (the integral $\mathcal{I}_{1}$ of Eq. (19)), whereas individual clump properties would mostly affect our estimates of its variance at short scales. For example, the clump number distribution is very likely to be cut off inside the galactic bulge because of strong tidal interactions with stars (Berezinsky et al. 2006). However, we do not expect that the results presented here would be strongly affected by these effects. For instance, we have shown that the steep energy dependence of the mean boost factor, in the case of a positron line, was mostly due to contributions from our very local environment, due to the short scale of positron propagation. A cut-off in the clump distribution for galactic radii less than $\sim 3 \mathrm{kpc}$ (corresponding to a minimum of $5 \mathrm{kpc}$ from the Earth) would then significantly diminish its low energy contribution, and would thus increase the relative variation of the boost factor with energy.

Cumberbatch \& Silk: It has been proposed recently (Cumberbatch \& Silk 2006) that the positron excess observed by HEAT could be due to the presence of a single clump located near the Earth. However, the situation in which the signal due to one clump dominates over the background due to all the others is very unlikely. The probability that a clump lies in the close proximity of Earth is sizeable only if the density of clumps is high, which in turn implies that many of them contribute to the measured flux. We showed that the quantitative study of this situation leads to unreasonably small probabilities.

Acknowledgements. We thank the PNC (French Programme National de Cosmologie) for financial support. J.L. is grateful to Alain Falvard and LPTA-Montpellier, and to Paschal Coyle and the ANTARES group at CPPM. J.P. thanks Jacques Colas and LAPP for extra financial support. Part of this work was discussed during the GdR-susy-PCHE meetings. We also thank the anonymous referee for thorough work on the first version of this paper and very helpful comments.

\section{References}

Agashe, K., \& Servant, G. 2004, Phys. Rev. Lett., 93, 231805

AMS Collaboration 2006, http://ams.cern.ch/

Baltz, E. A., \& Edsjö, J. 1999, Phys. Rev. D, 59, 023511

Barwick, S. W., Beatty, J. J., Bhattacharyya, A., et al. 1997, ApJ, 482, L191

Berezinsky, V., Dokuchaev, V., \& Eroshenko, Y. 2003, Phys. Rev. D, 68, 103003

Berezinsky, V., Dokuchaev, V., \& Eroshenko, Y. 2006, Phys. Rev. D, 73, 063504

Bertone, G., Hooper, D., \& Silk, J. 2004, Phys. Rep., 405, 279

Bertone, G., Zentner, A. R., \& Silk, J. 2005, Phys. Rev. D, 72, 103517

Boehm, C., Fayet, P., \& Schaeffer, R. 2001, Phys. Lett. B, 518, 8

Coutu, S., Beach, A. S., Beatty, J. J., et al. 2001, in International Cosmic Ray Conference, 1687

Cumberbatch, D., \& Silk, J. 2006 [arXiv: astro-ph/0602320]

Diemand, J., Moore, B., \& Stadel, J. 2005, Nature, 433, 389

Hofmann, S., Schwarz, D. J., \& Stöcker, H. 2001, Phys. Rev. D, 64, 083507

Hooper, D., \& Kribs, G. D. 2004, Phys. Rev. D, 70, 115004

Hooper, D., Taylor, J. E., \& Silk, J. 2004, Phys. Rev. D, 69, 103509

Maurin, D., \& Taillet, R. 2003, A\&A, 404, 949

Moore, B., Diemand, J., Stadel, J., \& Quinn, T. 2005

Moskalenko, I. V., \& Strong, A. W. 1998, ApJ, 493, 694

PAMELA collaboration 2006, http://wizard.roma2. infn.it/pamela/

Profumo, S., Sigurdson, K., \& Kamionkowski, M. 2006, Phys. Rev. Lett., 97, 031301

Servant, G., \& Tait, T. M. P. 2003, Nucl. Phys., B650, 391

Sjöstrand, T., Edén, P., Friberg, C., et al. 2001, Computer Physics Commun, 135, 238

Spergel, D. N., et al. 2006 [arXiv: astro-ph/0603449]

Zhao, H., Taylor, J., Silk, J., \& Hooper, D. 2005a [arXiv: astro-ph/0502049]

Zhao, H., Taylor, J., Silk, J., \& Hooper, D. 2005b [arXiv:astro-ph/0508215] 